\title{
CORONAL RAIN IN MAGNETIC ARCADES: REBOUND SHOCKS, LIMIT CYCLES, AND SHEAR FLOWS
}

\author{
X. Fang, C. Xia, R. Keppens, and T. Van Doorsselaere \\ Centre for Mathematical Plasma Astrophysics, Department of Mathematics, KU Leuven, Celestijnenlaan 200B, 3001 Leuven, Belgium \\ Received 2014 December 5; accepted 2015 April 21; published 2015 July 9
}

\begin{abstract}
We extend our earlier multidimensional, magnetohydrodynamic simulations of coronal rain occurring in magnetic arcades with higher resolution, grid-adaptive computations covering a much longer ( $>6 \mathrm{hr}$ ) time span. We quantify how blob-like condensations forming in situ grow along and across field lines and show that rain showers can occur in limit cycles, here demonstrated for the first time in 2.5D setups. We discuss dynamical, multi-dimensional aspects of the rebound shocks generated by the siphon inflows and quantify the thermodynamics of a prominencecorona transition-region-like structure surrounding the blobs. We point out the correlation between condensation rates and the cross-sectional size of loop systems where catastrophic cooling takes place. We also study the variations of the typical number density, kinetic energy, and temperature while blobs descend, impact, and sink into the transition region. In addition, we explain the mechanisms leading to concurrent upflows while the blobs descend. As a result, there are plenty of shear flows generated with relative velocity difference around $80 \mathrm{~km} \mathrm{~s}^{-1}$ in our simulations. These shear flows are siphon flows set up by multiple blob dynamics and they in turn affect the deformation of the falling blobs. In particular, we show how shear flows can break apart blobs into smaller fragments, within minutes.
\end{abstract}

Key words: magnetohydrodynamics(MHD) - Sun: corona - Sun: filaments, prominences

\section{INTRODUCTION}

Observations show recurrent formation and reshuffling of cool and dense material in coronal loops. The small scale $(\mathcal{O}(100) \mathrm{km})$ coronal rain is observed as cold, dense elongated blob-like features condensing in a much hotter loop and descending along one of its legs. The rain is guided by the loop magnetic field (Beckers 1962), dropping from heights of tens of Mm into the chromosphere (Kawaguchi 1970; Leroy 1972). Similar phenomena have been observed by analyzing absorption profiles in EUV spectral lines (Schrijver 2001; O'Shea et al. 2007). Seen to propagate from the top of the loop toward its footpoints (De Groof et al. 2004), systematic intensity variations in EUV spectral lines are confirmed as downflows of cool plasma, rather than representing slow magneto-acoustic waves (De Groof et al. 2005). Besides downflows toward footpoints, Tripathi et al. (2009) also found upflows toward the loop apex. Antolin et al. (2010) observed and tracked coronal rain in 30 active region loops and found more than one hundred descending condensations within 71 minutes. Tracing the cool material toward loop footpoints, Kamio et al. (2011) observed propagating patterns, suggesting a hot upflow following the downflows, supplying hot plasma into the loops. Antolin \& Rouppe van der Voort (2012) suggested that coronal rain consists of plenty of small blobs, with sizes around $300 \mathrm{~km}$ in width and $700 \mathrm{~km}$ in length on average and they also estimated the occurrence rate of coronal rain in active region loops to be once every two days. Since the solar corona is swamped with magnetic loops, this suggests a scenario where coronal rain is rather common.

Considering the very small sizes involved, one of the most attractive features of coronal rain is that it can be used to probe the local magnetic field structure, or that it can expose valuable properties of the local thermodynamic conditions inside coronal loops (Antolin et al. 2010). Indeed, the magnetic field structure has a much longer lifetime than the timescale for condensations to form and fall (Beckers 1962). Additionally, due to the low temperature (of order $10^{4} \mathrm{~K}$ ) of these condensations, coronal rain is normally observed in cold chromospheric lines (Levine \& Withbroe 1977; Müller et al. 2005). Coronal rain results from thermal instability, with its nonlinear counterpart and evolution also known as thermal non-equilibrium or catastrophic cooling. The linear thermal instability takes places whenever radiative losses locally overcome the heating input and is governed by well-known stability criteria for uniform radiative plasma conditions (Parker 1953; Field 1965). These can be met in the coronal temperature range, as one encounters locally negative slopes in the radiative loss function $\Lambda(\mathrm{T})$ as a function of temperature. When thermal conduction is insufficient in transporting enough energy to cooling (and condensing) material, the temperature reduces over time. As a consequence of thermal instability, temperature and gas pressure drop dramatically in the perturbed region, resulting in matter sucked in from the surroundings to the perturbed region, forming an increasingly larger and cooler condensation. This runaway effect will continually increase the density and decrease the temperature of condensations until heating and cooling achieve a balance again at lower temperatures and higher densities. Numerical simulations have contributed to our understanding of thermal instability over the last 40 years (Goldsmith 1971; Hildner 1974; Mok et al. 1990, 2008; Antiochos \& Klimchuk 1991; Dahlburg et al. 1998; Antiochos et al. 1999, 2000; Karpen et al. 2003, 2006; Karpen \& Antiochos 2008; Xia et al. 2011). Early numerical work shows that in the million-degree solar corona, small temperature contrasts could be enhanced by line and recombinationdriven radiative losses within several minutes (Goldsmith 1971). Catastrophic cooling drives recombination of elements in the cool condensations, making them partially ionized and visible in cool chromospheric lines. Hildner (1974) concluded that the rate of condensation is mainly determined by hydrodynamical processes.

Important progress in modeling was achieved by Antiochos \& Klimchuk (1991) using a spatially dependent heating increase that is localized closer to the chromospheric footpoints 
than to the loop midpoint. With this localized heating at the footpoints, Dahlburg et al. (1998) pointed out that another key requirement to generate a stable, prominence-like condensation is a dipped geometry in the loop. With an adaptive grid code, Antiochos et al. (1999) showed, in a one-dimensional (1D) model, that the complete growth of condensation reached a quasi-steady state after $\approx 5000 \mathrm{~s}$. In a similar $1 \mathrm{D}$ setup, Xia et al. (2011) calculated the linear instability criterion from numerical results and proved that the onset of coronal condensation indeed satisfies the linear isochoric instability criterion (Parker 1953). In the solar corona, the fairly high densities required for the instability onset are thought to be obtained by evaporating material with heating located near the footpoints of coronal loops in the chromosphere or by direct mass injection into the corona (Wang 1999; Chae et al. 2001), resulting, e.g., from nano-heating events.

Influenced by magnetohydrodynamic forces (gravity, Lorentz force, and gas pressure gradients), condensations, once formed, either fall from the corona down to the chromosphere as coronal rain or they collect into larger structures and remain suspended in the corona over long time periods as prominences, supported by the magnetic field. Many numerical works addressed formation and dynamics of coronal rain, but adopted simplifying 1D approximations, reducing the problem to gas dynamic, thermodynamic evolutions along individual field lines (Antiochos \& Klimchuk 1991; Karpen et al. 2001, 2005; Schrijver 2001; Müller et al. 2003, 2004; De Groof et al. 2005; Mendoza-Briceño et al. 2005; Karpen et al. 2006; Antolin et al. 2010; Xia et al. 2011; Luna et al. 2012). Since coronal rain occurs in many active region loops, the heating input is generally thought to be concentrated at the loop footpoints (Antiochos \& Klimchuk 1991; Mendoza-Briceño et al. 2005), evaporating chromospheric plasma into the loops and increasing the density. With persistent heating, the anisotropic thermal conduction and optically thin radiation lead these coronal hot loops to reach thermally unstable regimes with a higher density in a timescale of hours (Xia et al. 2011). Then catastrophic cooling sets in locally, resulting in the fast formation of cool condensations, as demonstrated in 1D models (Karpen et al. 2001; Müller et al. 2003; De Groof et al. 2005; Karpen et al. 2005). Numerical simulations by Müller et al. (2004) emphasized that a loss of equilibrium at the loop apex and the process of catastrophic cooling is caused by constant heating concentrated at the footpoints of the loop rather than a drastic decrease of the total loop heating which was used in earlier models. Müller et al. (2005) compared observations from an EIT shutterless campaign with simulations of coronal loops and confirmed that observed localized brightenings and fast flows are consistent with this model. An important conclusion from Antolin et al. (2010) was that the structure and dynamics of the coronal rain blobs are more sensitive to the pressure variations arising from catastrophic cooling than to gravity itself. This is in agreement with Schrijver (2001), who suggested that the internal pressure evolution of the loops, rather than gravity, determines the condensation speeds. Furthermore, Antolin et al. (2010) indicated that if a loop is predominantly heated by Alfvén waves, coronal rain is inhibited since they tend to heat the loop uniformly. Hence, coronal rain may not only point to the spatial distribution of the heating in coronal loops but also to the agent of the heating itself. They thus propose coronal rain as a marker for coronal heating mechanisms. Xia et al. (2011) pointed out that steady heating is not necessary to sustain the condensation. Once the condensation is formed, it keeps growing even after localized heating ceases. Luna et al. (2012) simulated a threedimensional (3D) sheared double arcade with a large ensemble of $1 \mathrm{D}$ independent flux tubes and observed the formation of both prominence threads and coronal rain.

Recently, Fang et al. (2013) presented the first multidimensional, magnetohydrodynamic simulations which captured the initial formation and the long-term sustainment of the coronal rain phenomenon. There we found that coronal rain in arcades is always accompanied by fast counter-streaming siphon flows in neighboring flux bundles and we statistically analyzed 80 minutes of virtual coronal rain in terms of sizes, mass, and velocity patterns. Our 2.5D simulations showed how blobs deform into $\mathrm{V}$-shaped patterns, and had blobs that levitate, evaporate in situ, or fall into the transition region (TR) at speeds below free-fall. IRIS data recently also revealed many coronal rain impact events, with up to supersonic speeds above sunspots (Kleint et al. 2014). We therefore revisited our MHD setup from Fang et al. (2013), at even further increased numerical resolution and for much longer time, going up to $6 \mathrm{hr}$ in total. We now analyze blob formation and blob impact in more detail, focusing on multi-dimensional aspects not probed by 1D setups. Furthermore, the High-resolution Coronal Imager (Hi-C) in 2013 July provided a much more detailed look at the fine structure and dynamics in the solar corona. With data from Hi-C, Alexander et al. (2013) reported that anti-parallel flows have been directly imaged along fundamental filament threads within the million degree corona. They measured relative flow velocities of similar magnitude as in our previous simulations, namely $70-80 \mathrm{~km} \mathrm{~s}^{-1}$. Both observations and our simulations hence suggest that such counter-streaming flows are likely commonplace. We observed that siphon flows establish naturally in a raining arcade, with velocity differences on adjacent field lines up to $80 \mathrm{~km} \mathrm{~s}^{-1}$. We thus also extended our simulations to further argue how our setup in a low field (order $12 \mathrm{G}$ ) magnetic arcade relates to the observed clumps of falling coronal rain (Antolin et al. 2010) and to unresolved fine-scale structure in solar coronal loop-tops (Scullion et al. 2014).

The paper is then organized as follows: in Section 2 we describe the numerical setup, in Section 3.1 we describe the multidimensional aspects of the condensations, focusing on rebound shocks and their Prominence-Corona-Transion Region (PCTR) structure, Section 3.2 discusses the condensation rates and the long term coronal rain limit cycle obtained, Section 3.3 quantifies blob impact on the TR and concurrent upflows, in Section 3.4 we investigate the counter-streaming flows, and in Section 3.5 we describe the shear flow effects. Conclusions are drawn in Section 4.

\section{COMPUTATIONAL ASPECTS}

\subsection{Governing Equations and Initial Setup}

Our numerical setup follows our previous 2.5D thermodynamic MHD simulation from Fang et al. (2013), which includes gravity, field-aligned heat conduction, and radiative cooling and parametrized heating terms, on a rectangular plane with horizontal extension $-40 \mathrm{Mm} \leqslant x \leqslant 40 \mathrm{Mm}$ and vertical extension $0 \leqslant y \leqslant 50 \mathrm{Mm}$. The governing equations are as 
follows:

$$
\begin{gathered}
\frac{\partial \rho}{\partial t}+\nabla \cdot(\rho \boldsymbol{v})=0, \\
\frac{\partial(\rho \boldsymbol{v})}{\partial t}+\nabla \cdot\left(\rho \boldsymbol{v} \boldsymbol{v}+p_{\mathrm{tot}} \boldsymbol{I}-\frac{\boldsymbol{B} \boldsymbol{B}}{\mu_{0}}\right)=\rho \boldsymbol{g}, \\
\frac{\partial E}{\partial t}+\nabla \cdot\left(E \boldsymbol{v}+p_{\mathrm{tot}} \boldsymbol{v}-\frac{\boldsymbol{v} \cdot \boldsymbol{B}}{\mu_{0}} \boldsymbol{B}\right) \\
=\rho \boldsymbol{g} \cdot \boldsymbol{v}+\nabla \cdot(\boldsymbol{\kappa} \cdot \nabla T)-Q+H, \\
\frac{\partial \boldsymbol{B}}{\partial t}+\nabla \cdot(\boldsymbol{v} \boldsymbol{B}-\boldsymbol{B} \boldsymbol{v})=0,
\end{gathered}
$$

where $T, \rho, \boldsymbol{B}, \boldsymbol{v}$, and $\boldsymbol{I}$ are respectively temperature, density, magnetic field, velocity, and unit tensor, with the ratio of specific heats $\gamma=5 / 3$, and a total energy density of $E=p /(\gamma-1)+\rho v^{2} / 2+B^{2} / 2 \mu_{0} ; p_{\text {tot }} \equiv p+B^{2} / 2 \mu_{0}$ is the total pressure, consisting of magnetic pressure and thermal pressure $p ; \boldsymbol{g}=g_{0} R_{\odot}^{2} /\left(R_{\odot}+y\right)^{2} \hat{y}$ is the gravitational acceleration with the solar surface gravitational acceleration $g_{0}=-274 \mathrm{~m} \mathrm{~s}^{-2}$ and the solar radius $R_{\odot} ; H$ and $Q$ are respectively the heating and radiative loss rates; and $\boldsymbol{\kappa}$ is the thermal conductivity tensor. Assuming a 10:1 abundance of hydrogen and helium of completely ionized plasma, we obtain $\rho=1.4 m_{p} n_{\mathrm{H}}$, where $m_{p}$ is the proton mass and $n_{\mathrm{H}}$ is the number density of hydrogen. We use the ideal gas law $p=2.3 n_{\mathrm{H}} k_{\mathrm{B}} T$, where $k_{\mathrm{B}}$ is the Boltzmann constant. We also adopt $Q=1.2 n_{\mathrm{H}}^{2} \Lambda(T)$ as the radiative cooling term, where $\Lambda(T)$ is the radiative loss function for optically thin emission, quantified by Colgan et al. (2008) using a recommended set of quiet-region element abundances, as used in our previous work (Xia et al. 2011, 2012; Fang et al. 2013; Keppens \& Xia 2014). In the calculations, Colgan et al. (2008) used a complete and self-consistent atomic data set and an accurate atomic collisional rate over a wide temperature range. Below $10,000 \mathrm{~K}$, we set $\Lambda(T)$ to vanish because the plasma there is optically thick and no longer fully ionized. We use the exact integration method introduced by Townsend (2009) to evaluate the radiative loss term. The use of explicit, (semi-)implicit, and exact integration methods in grid-adaptive simulations has been compared in van Marle et al. (2011). The term containing $\boldsymbol{\kappa}=\kappa_{||} \hat{\boldsymbol{b}} \hat{\boldsymbol{b}}$ quantifies the anisotropic thermal conduction along the magnetic field lines, composed of the unit vector $\hat{\boldsymbol{b}}$ along the magnetic field and the Spitzer conductivity $\kappa_{||}$as $10^{-6} T^{5 / 2}$ $\operatorname{erg~} \mathrm{cm}^{-1} \mathrm{~s}^{-1} \mathrm{~K}^{-3.5}$.

We employ a linear force-free magnetic field for the initial magnetic configuration, which is characterized by a constant angle $\theta_{0}$ as follows:

$$
\begin{aligned}
& B_{x}=-B_{0} \cos \left(\frac{\pi x}{L_{0}}\right) \sin \theta_{0} \exp \left(-\frac{\pi y \sin \theta_{0}}{L_{0}}\right), \\
& B_{y}=B_{0} \sin \left(\frac{\pi x}{L_{0}}\right) \exp \left(-\frac{\pi y \sin \theta_{0}}{L_{0}}\right), \\
& B_{z}=-B_{0} \cos \left(\frac{\pi x}{L_{0}}\right) \cos \theta_{0} \exp \left(-\frac{\pi y \sin \theta_{0}}{L_{0}}\right),
\end{aligned}
$$

with $\theta_{0}=30^{\circ}$; the arcade makes a $30^{\circ}$ angle with the neutral line $(x=0, y=0) . L_{0}=80 \mathrm{Mm}$ is the horizontal size of our domain from -40 to $40 \mathrm{Mm}$, and when adopting $B_{0}=12 \mathrm{G}$, our magnetic arcade has a total box averaged field strength of $2.9 \mathrm{G}$.

For the initial thermal structure, we set a uniform temperature of $10,000 \mathrm{~K}$ below a height of $2.7 \mathrm{Mm}$ and choose a temperature profile with height ensuring a constant vertical thermal conduction flux (i.e., $\kappa \partial T / \partial y=$ $2 \times 10^{5} \mathrm{erg} \mathrm{cm}^{-2} \mathrm{~s}^{-1}$ ) above this height, as also exploited by other authors (Fontenla et al. 1991; Mok et al. 2005). The initial density is then derived by assuming hydrostatic equilibrium with the number density of $1.2 \times 10^{15} \mathrm{~cm}^{-3}$ at the bottom and the initial velocity field of all plasma is static. Since the corona needs to achieve a self-consistent thermal structure, we employ a background heating rate decaying exponentially with height into the whole system all the time,

$$
H_{0}=c_{0} \exp \left(-\frac{y}{\lambda_{0}}\right),
$$

where $c_{0}=10^{-4} \mathrm{erg} \mathrm{cm}^{-3} \mathrm{~s}^{-1}$ and $\lambda_{0}=50 \mathrm{Mm}$. This heating is meant to balance the radiative losses and heat conduction related losses of the corona in its steady state. The slight difference in heating scale height between $50 \mathrm{Mm}$ in Equation (6) above and $36 \mathrm{Mm}$ in Equation (2) in Fang et al. (2013) improves numerical stability at the top boundary and prevents it from cooling down during the longer timescale run performed here. With the above initial setup the whole system is now out of thermal equilibrium. We integrate the governing equations in time with heating $H=H_{0}$ active until the system achieves a quasi-equilibrium state. After 72 minutes, the above configuration reaches a quasi-equilibrium state shown in Figure 1, which represents a $3 \mathrm{D}$ impression of the numerical box quantifying the temperature and number density profile and selected magnetic field lines. The $t=0$ in Figure 1 means that after reaching the quasi-equilibrium state, we reset the time of the system back to zero for the next stage of simulation. As seen in Figure 1, the numerical relaxation phase leads to some thermodynamic structuring in the final arcade. Some chromospheric plasma is quickly evaporated into coronal loops at the beginning of the relaxation, but this material gradually loses its kinetic energy. As a result, the final relaxed state of the system is identified as the time when the maximal residual velocity in the simulation is less than $5 \mathrm{~km} \mathrm{~s}^{-1}$. In this end state, Figure 1 shows a relatively thin TR located at heights between 3 and $5 \mathrm{Mm}$, which connects the chromosphere to corona. This TR is higher above the neutral line, due to less downward thermal flux there because of the strong horizontal magnetic field. The plasma beta is 0.06 at $20 \mathrm{Mm}$ height above the neutral line while the temperature and number density there are around $1.7 \mathrm{MK}$ and $3.5 \times 10^{8} \mathrm{~cm}^{-3}$. The total mass (per unit length in the ignored dimension) of hot plasma in the corona is around $3.2 \times 10^{4} \mathrm{~g} \mathrm{~cm}^{-1}$.

Following this equilibrated system, we turn on a relatively strong heating $H_{1}$. This extra heating is localized near 


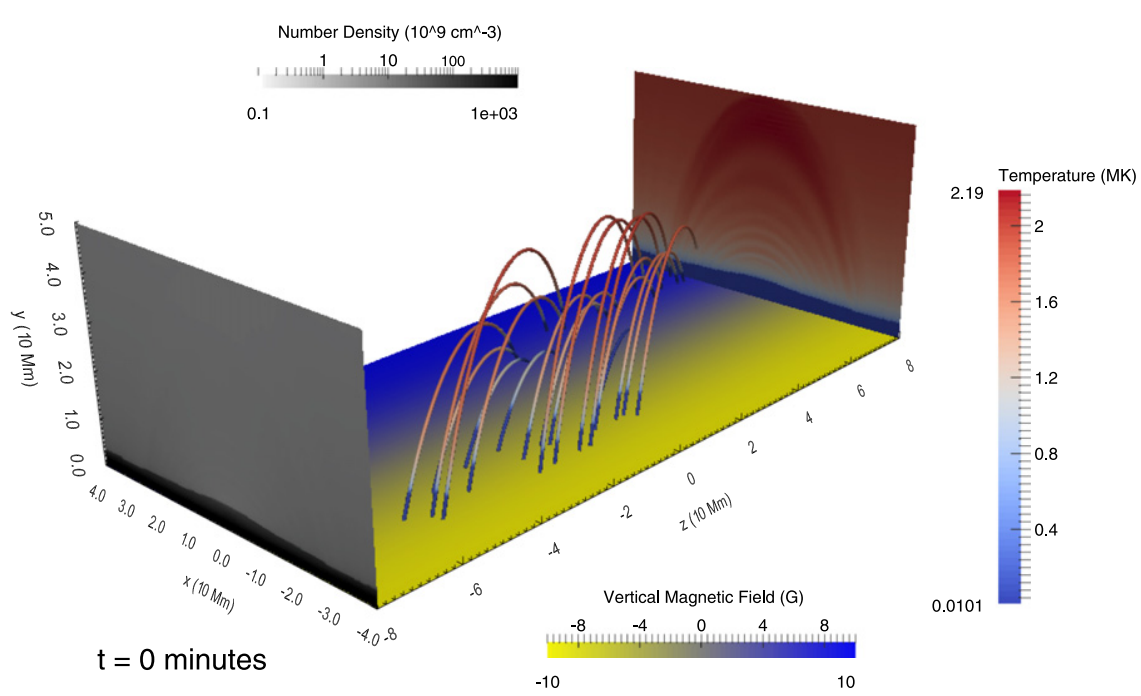

Figure 1. Around $t \approx 70$ minutes after relaxation, we show a 3D view on the quasi-equilibrium stage of our simulation, which serves as the initial condition when extra localized heating is turned on. ( $t=0$ minutes means the resetting of time to zero from now on.) The randomly selected field lines are colored by temperature; the back cross-section shows the temperature while the front $x-y$ cross-section shows the number density map.

footpoints in the chromosphere with formula (Fang et al. 2013):

$$
\begin{gathered}
H_{1}=\left\{\begin{array}{l}
c_{1} \quad \text { if } y<y_{c} \\
\text { and } A\left(x_{1}, 0\right)<A(x, y)<A\left(x_{2}, 0\right) \\
c_{1} \exp \left(-\left(y-y_{c}\right)^{2} / \lambda^{2}\right) \\
\text { and } A\left(x_{1}, 0\right)<A(x, y)<A\left(x_{2}, 0\right)
\end{array} \text { if } y \geqslant y_{c}\right.
\end{gathered}
$$

where $c_{1}=10^{-2} \mathrm{erg} \mathrm{cm}^{-3} \mathrm{~s}^{-1}, \quad y_{c}=3 \mathrm{Mm}, \quad x_{1}=26 \mathrm{Mm}$, $x_{2}=14 \mathrm{Mm}, a=0.8 \mathrm{Mm}^{2}$, and $b=1.2 \mathrm{Mm}^{2}$. This choice of strong base heating contrast $\left(c_{1} / c_{0}=100\right)$ between $H_{1}$ and $H_{0}$ can mimic flare related chromospheric evaporation. However, this $H_{1}$ heating decreases with height to very small values and reaches one-tenth of $H_{0}$ heating at $10 \mathrm{Mm}$. As a result, the $H_{1}$ heating dominates heating in the chromosphere and the TR, while the $H_{0}$ heating plays a more important role in the heating in the corona. The parameter $y_{c}=3 \mathrm{Mm}$ represents the height of the TR in the quasi-equilibrium system. $A(x, y)$ is the magnetic potential depending on the location and decaying exponentially with height into the whole system. Because the magnetic potential along a single magnetic field line is constant, we add extra heating at both feet of all magnetic field lines identified by $A(x, y)$ in the range of $x_{1}<|x|<x_{2}$. Since catastrophic cooling is very sensitive to the heating decay scale and the length of magnetic field lines (Xia et al. 2011), the heating decay scale $\lambda$ is set to larger values for longer field lines by the above formulae.

\subsection{Discretization, AMR Settings and Boundary Treatment}

We use the MPI-parallelized Adaptive Mesh Refinement (AMR) Versatile Advection Code MPI-AMRVAC (Keppens et al. 2012; Keppens \& Porth 2014; Porth et al. 2014) to run the simulation. An effective resolution of $4096 \times 2560$ or an equivalent spatial resolution of $20 \mathrm{~km}$ in both directions is obtained through six AMR levels. This represents an effective fourfold improvement in resolution with respect to our earlier model (Fang et al. 2013). Our numerical strategy to advance the governing partial differential equations uses a three-step Runge-Kutta type scheme. For flux computations, a thirdorder-accurate limited reconstruction (Čada \& Torrilhon 2009) is introduced to calculate the variable evaluation from cell center to cell edge. We adopt a suitably mixed prescription between a diffusive total variation diminishing Lax-Friedrichs and contact-resolving Harten-Lax-van Leer with contact restored (HLLC) scheme (Meliani et al. 2008).

For the boundary treatment, we employ two grid layers exterior to the domain as ghost cells to prescribe cell center values. Considering the left and right physical boundary, density, energy, $y$ and $z$ momentum components, $B_{y}$ and $B_{z}$ are set symmetrically, while $v_{x}$ and $B_{x}$ are adopted antisymmetrically to ensure zero face values. In the bottom boundary ghost cells, we use the primitive variables $(\rho, v, p, \boldsymbol{B})$ to set all velocity components antisymmetrically to enforce both noflow-through (vertical) and no-slip (horizontal), while the $\boldsymbol{B}$ are fixed to the initial analytic expressions of Equation (5), and the stratification of density is kept at pre-determined values from the initial condition, as well as the pressure. We always resolve the bottom region up to $y=0.5 \mathrm{Mm}$ at the maximum resolution. For the top conditions, we set all velocity components as antisymmetric, and adopt a discrete pressuredensity extrapolation from the top layer pressure with a maximal temperature $T_{\text {top }}=2 \times 10^{6} \mathrm{~K}$. For the magnetic field, we use a two-cell zero-gradient extrapolation to determine $\boldsymbol{B}$ in the ghost cells and improve $B_{y}$ from a second order one-sided centered difference evaluation of $\nabla \cdot \boldsymbol{B}=0$.

\section{RESULTS AND DISCUSSION}

In our previous work (Fang et al. 2013), we already described the formation process for the first condensation and emphasized how it perturbed the overall force balance in a 2D fashion. In this work, we discuss more of the multi-dimensional 


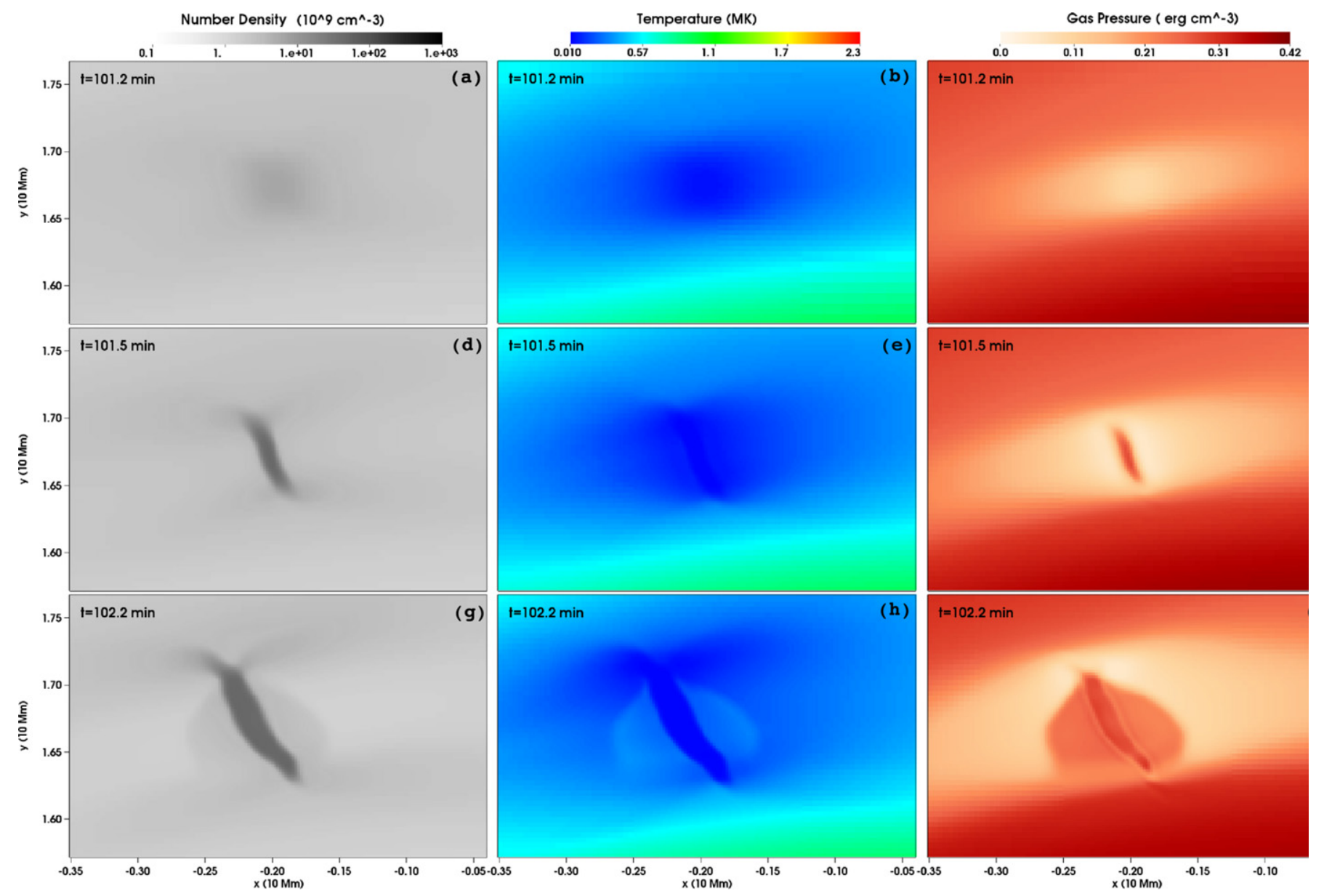

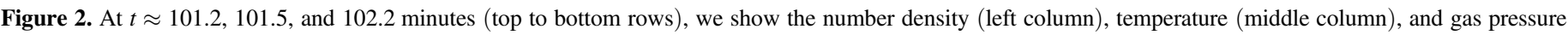
(right column) maps in a zoomed (about $3000 \times 2000 \mathrm{~km}^{2}$ ) area. This shows the formation process of the first condensation.

details for the forming condensations, and compare the results of our 2.5D simulations with previous 1D simulation works (Müller et al. 2003; Xia et al. 2011) and insights from observations (Antolin \& Rouppe van der Voort 2012), in particular paying attention to the cross-field effects.

\subsection{Rebound Shocks and PCTR of Condensations}

The forming process of the first condensation in our 2.5D simulation is shown in Figure 2, which presents the temporal evolution of number density (left columns), temperature (middle columns), and gas pressure (right columns) at $t \approx$ $101.2,101.5$, and 102.2 minutes. When we compare these results with the corresponding Figures 5 and 7 of 1D hydrodynamic simulations in Xia et al. (2011), we conclude that all three parameters behave similarly in the forming process, as the number density increases rapidly from $10^{8} \mathrm{~cm}^{-3}$ to $10^{10} \mathrm{~cm}^{-3}$, while the temperature decreases down to $0.01 \mathrm{MK}$. Along each arched field line, this is analogous to the sudden thermal instability onset in 1D runs. This similarity confirms the applicability of restricted $1 \mathrm{D}$ model efforts that assume a rigid 1D loop under the prevailing plasma $\beta$ conditions, which takes on a local value of around 0.06. The middle panel in the right column of Figure 2 also shows significantly increased gas pressure inside the condensation and a layer of low gas pressure surrounding it after its formation. In the bottom panels of Figure 2, we notice that density, temperature, and gas pressure all reveal a front propagating as expanding wings on both sides of the condensation. This phenomenon is because fast siphon inflows are driven into the forming condensation by a strong pressure gradient between the lower gas pressure around the condensation and relatively higher gas pressure away from the condensation, as seen in the middle panel in the right column of Figure 2. These two siphon flows meet up with the blobs and dynamically impact on the blob to generate two rebound shocks. Hence, while thermal instability and runaway cooling triggers a growing condensation, one also forms two rebound shock fronts that propagate away from the blob. The slightly different formation times at different parts of the condensation on adjacent magnetic field lines (Fang et al. 2013), which are due to gradual variations in length and chromospheric footpoint conditions, are the reason that these two expansion shock fronts display a fan-shaped structure, forming earliest in the blob center and spreading away from the blob. This fan-shaped structure of the rebound shocks is also clearly observed in Xia et al. (2012).

However, not every condensation realizes this nearly leftright symmetric situation as seen near the loop apex for this first condensation from Figure 2. Due to slightly asymmetric conditions already prevailing after the numerical relaxation process and due to perturbations from existing condensations, most of the following condensations initiate in loop limbs (also shown in the online movies of Fang et al. 2013). The fieldprojected gravity force on the limbs leads to asymmetric 

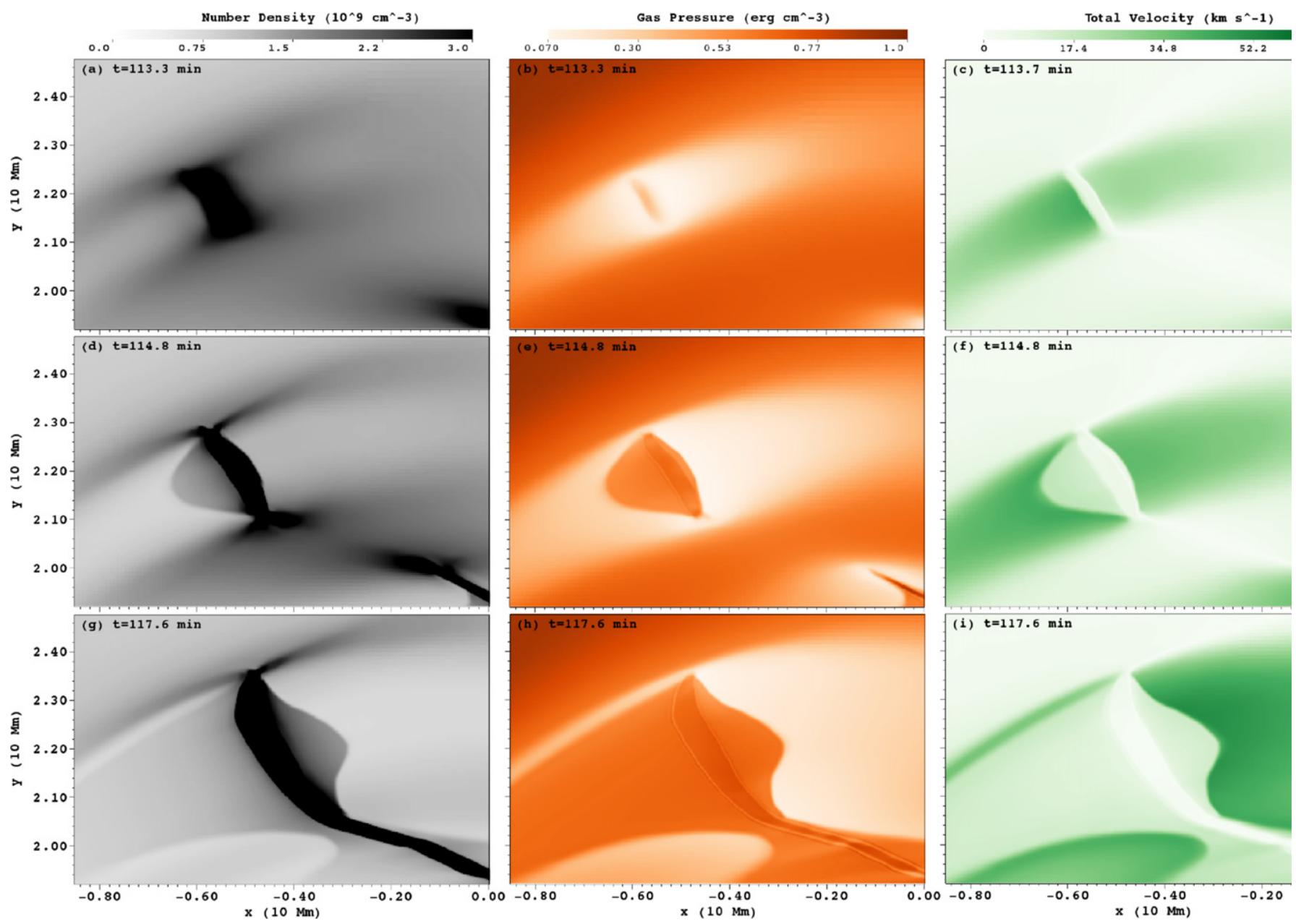

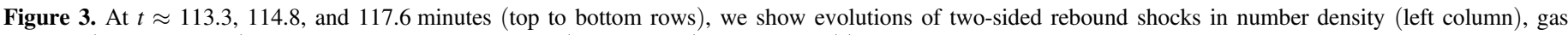

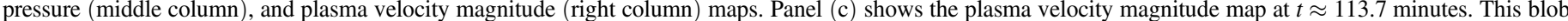
shows clear left-right asymmetric behaviour in its rebound shock pair pattern.

plasma distributions, as seen, e.g., in the number density map in panel (a) of Figure 3 at $t \approx 113.3$ minutes, the moment when local catastrophic cooling begins there (about 10 minutes after the first condensation). The higher central gas pressure indicates the initial forming location of this condensation in panel (b) of Figure 3. Due to its limb-loop location, the number density map points out that the right side of the condensation holds a relatively denser $\left(3 \times 10^{9} \mathrm{~cm}^{-3}\right)$ and wider plasma distribution than the left $\left(1.5 \times 10^{9} \mathrm{~cm}^{-3}\right)$. Still, strong pressure gradients drive siphon flows from both sides toward this condensation. After a short time at $t \approx 113.7$ minutes, the denser and heavier plasma at the right of this condensation has a (left-directed) siphon flow with a slower speed of $23 \mathrm{~km} \mathrm{~s}^{-1}$, compared to the left siphon flow (which is right-directed) at a speed of $42 \mathrm{~km} \mathrm{~s}^{-1}$, shown by the velocity magnitude map in panel (c) of Figure 3. As discussed above, the impact of siphon flows on the condensation naturally generates rebound shocks, whose speeds are determined by the original speeds of the siphon flows and the mass contrast between the condensation and the siphon flows. The slower and heavier siphon flow on the right of the blob here leads to a much slower rebound (right-directed) shock seen to separate at $7 \mathrm{~km} \mathrm{~s}^{-1}$, while the left one (left-directed) travels at $21 \mathrm{~km} \mathrm{~s}^{-1}$. These two rebound shocks are identifiable in the gas pressure map in panel (e) of Figure 3 at $t \approx 114.8$ minutes. The condensation itself has a velocity of $5 \mathrm{~km} \mathrm{~s}^{-1}$, meaning that basically the right rebound shock barely can sweep up and heat little siphon flow plasma. Because the central condensation keeps sucking in plasma from nearby and the rebound shock at the right of the blob is too slow to sweep and heat up plasma, the gas pressure there does not rise to a higher value and keeps a strong pressure gradient at the right of the blob, as shown in panel (e) of Figure 3. About 3 minutes later at $t \approx 117.6$ minutes, this persistent pressure gradient at the right of the blob accelerates the leftdirected siphon flow to a higher speed of $52 \mathrm{~km} \mathrm{~s}^{-1}$ (shown in panel (i) of Figure 3); therefore, the corresponding rightdirected rebound shock finally speeds up to $28 \mathrm{~km} \mathrm{~s}^{-1}$ and begins to sweep and shock-heat the plasma on its way. In short, initial asymmetric situations on the condensation can lead to a complicated thermal and dynamical evolution and result in a delay of rebound shocks spreading at one side of the condensation.

Additionally, we also find another special case, namely blob A in Figure 4, which has only one rebound shock on its left side. Figure 4 shows the gas pressure map (a) and (b) at $t \approx 134.8$ and 137.6 minutes, with a dotted isocontour of the number density at $7 \times 10^{9} \mathrm{~cm}^{-3}$ overplotted. This density contour at $7 \times 10^{9} \mathrm{~cm}^{-3}$ is one of the criteria that identifies whether a cell contains cool plasma belonging to coronal rain, as used later on. Panel a in Figure 4 indicates a similar situation 


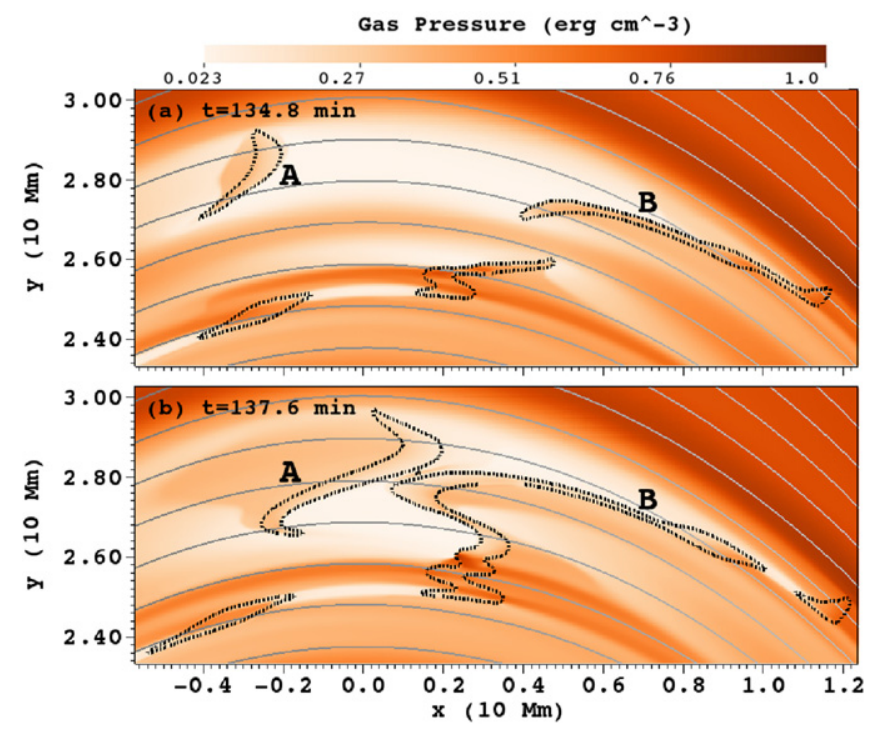

Figure 4. At $t \approx 134.8$ and 137.6 minutes, we show the gas pressure map (a) and (b) at times indicated, with a dotted isocontour of the number density at $7 \times 10^{9} \mathrm{~cm}^{-3}$. The thin gray lines are magnetic field lines. There are two blobs $\mathrm{A}$ and $\mathrm{B}$ in the same loop, with consequences for the way siphon flows can induce or prevent rebound shock patterns.

for blob $\mathrm{A}$ as in the second row of Figure 3 in which only the left rebound shock spreads out. In contrast to what happens in the third row of Figure 3, for blob A we do not find a right rebound shock in Figure 4 until the collision and merging of blob A with blob B. The reason is that when the thermal instability triggers the condensation labeled there as blob A, another existing condensation labeled as blob B in the same coronal loop has already depleted the plasma between these two blobs. Therefore the small pressure gradient in the emptied loop between the two blobs cannot drive a fast siphon flow to create a strong rebound shock for blob A, even though the gas pressure on the right of blob $\mathrm{A}$ is low enough (panel (a) in Figure 4). Afterwards, when blob A catches up and merges with blob $\mathrm{B}$ because of the strong pressure gradient outside these two blobs, the rebound shock at the right side of blob A is still not fast enough to show clear separation and propagation.

We also observe the details of a gas pressure substructure within these shock-bounded regions around the condensation in the simulations. These reveal the establishment of a PCTR-like structure around all blobs. The gas pressure substructure around the first condensation consists of three components shown in panel (a) of Figure 5 and panel (i) of Figure 2, namely a high gas pressure outside of the condensation, a low gas pressure at the boundary of the condensation, and a higher gas pressure in the center of the condensation. Actually, it is not only this first condensation in Figure 2 that has this kind of gas pressure substructure, but all the blobs which establish a dynamic equilibrium around themselves also have it, e.g., all the blobs in Figures 3 and 4. To better quantify this, we identify a field line crossing the center of the blob shown in panel (a) of Figure 5 and plot gas pressure, temperature and radiative loss along this field line in panel (c) of Figure 5. The temperature declines from a coronal temperature of $0.35 \mathrm{MK}$ to a cool plasma temperature of $0.01 \mathrm{MK}$ in $200 \mathrm{~km}$ and density increases from $1 \times 10^{8} \mathrm{~cm}^{-3}$ to $1 \times 10^{10} \mathrm{~cm}^{-3}$; therefore this $200 \mathrm{~km}$ area could basically be considered a PCTR. Within this area, we find that two highly radiative loss peaks exist, introduced by a temperature around $0.02 \mathrm{MK}$. This corresponds to the two dips of gas pressure at the boundary of the blob. These two strong radiation areas also indicate the location in which catastrophic cooling takes place, ensuring that the condensation keeps growing. Indeed, the two dips in gas pressure always relocate with the boundary of the blobs, coincident with the strong emissive loss. Although the temperature of $0.01 \mathrm{MK}$ inside the blob is lower than in the surrounding coronal plasma, a much higher density at the center of the condensation $\left(5 \times 10^{10} \mathrm{~cm}^{-3}\right)$ leads to a slightly higher gas pressure there. The high gas pressure outside of the condensation reflects the post shock conditions prevailing there after the rebound shocks run against the condensation inflows. Note that our resolution is such that we have about seven grid points along the field line through the PCTR at each side of the blob in Figure 5, clearly resolving the PCTR around the blob in our simulation.

The difference in gas pressure between the inside and outside of the condensation is found to persist throughout the lifetime of the blobs and plays a role in the movement of the blobs. Especially when the blobs fall along the field lines toward footpoints, the gas pressure and temperature ahead of the descending blob increase as shown in panel (b) of Figure 5 due to the blob compressing the plasma ahead of it in the loop and the strong evaporation at the loop footpoints. We also identify a field line crossing the center of the blob shown in panel (b) of Figure 5 and plot gas pressure, temperature, and radiation loss along this field line in panel (d) of Figure 5, which also shows an obvious PCTR. Due to the gravity variation and the strong gas pressure gradient between the two sides of the blob, the lower part of this blob has a higher density distribution, which naturally leads to a higher radiative loss. This strong gas pressure gradient slows down the acceleration of the blob in its descent. This was also pointed out in Fang et al. (2013), where we stated that sometimes, it can even lift lighter blobs to cross the loop apex.

\subsection{Coronal Rain Limit Cycle and Condensation Rate}

Panel (a) of Figure 6 shows the temporal evolution of the total mass of cool (solid) and hot (dashed) plasma in the corona, and panel (b) of Figure 6 shows the number of blobs for the entire time interval of our 2.5D simulation. The criteria to identify whether a cell contains cool plasma belonging to coronal rain are that (i) the number density is higher than $7 \times 10^{9} \mathrm{~cm}^{-3}$, (ii) the temperature is lower than $2 \times 10^{4} \mathrm{~K}$, and (iii) the location is above the chromosphere-corona-transitionregion. We dynamically locate the height of the TR at each $x$ position as $y^{\operatorname{tr}}(x, t)$ by searching the vertical position of the (first) maximum gradient value of temperature from the bottom boundary. Each blob is defined as a collection of neighboring cells which hold cool plasma. However, if the number of grid cells in one blob is smaller than 10 at our highest resolution, we remove this blob from the blob list to avoid counting spurious transient features that do not collect into a clearly resolvable blob, and also to mimic the observational resolution. As stated before, we adopt a four times higher resolution than in Fang et al. (2013), but also extend the simulation to a two times longer time of around 370 minutes (previously 190 minutes). By running our 2.5D simulation for these much longer times, we find that the whole coronal rain process shows limit cycles, which has been discussed in earlier 1D simulations (Müller et al. 2003), as well as in observational work (Antolin \& 

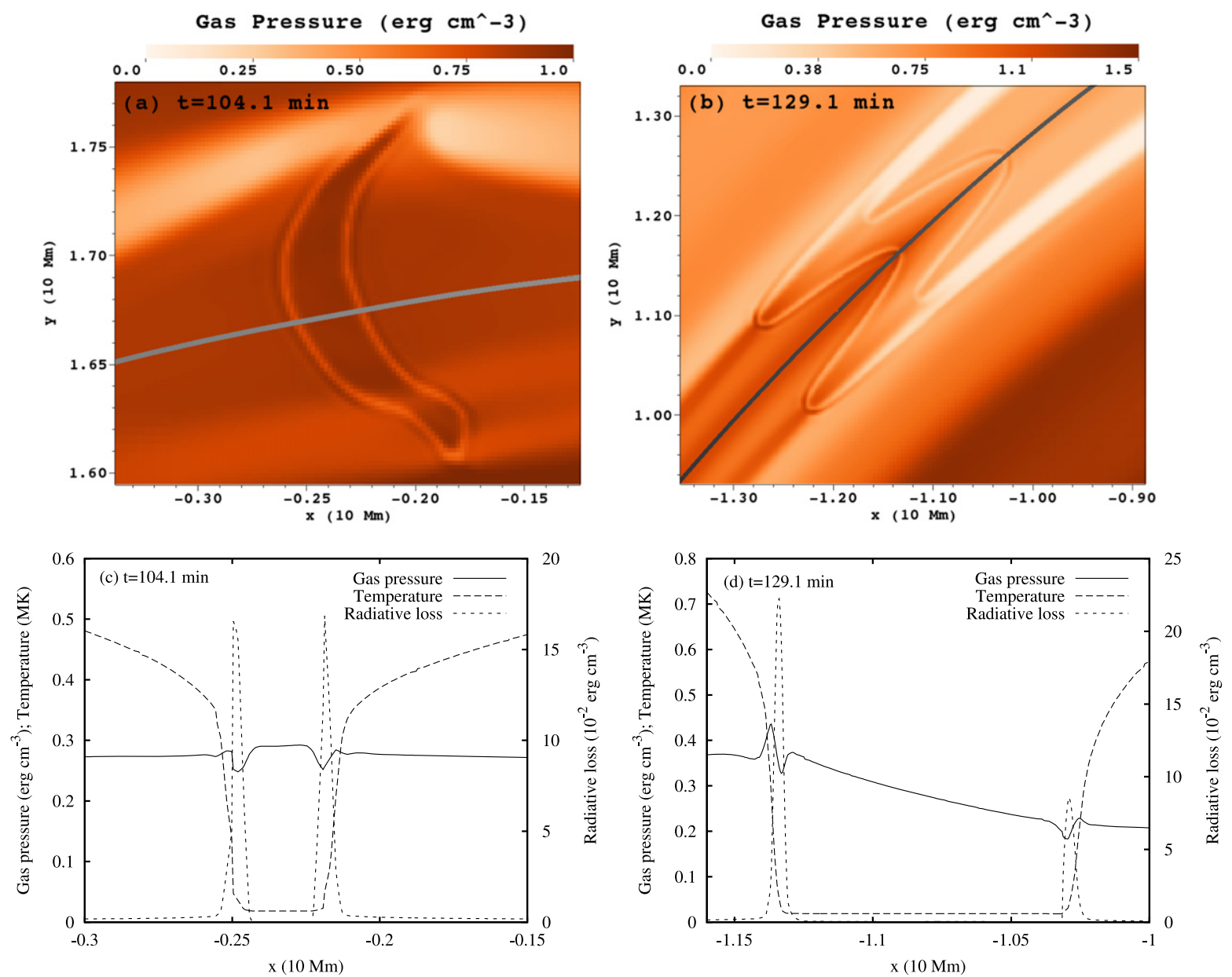

Figure 5. At $t \approx 104.1$ (left) and 129.1 minutes (right), we show in the top row panels (a) and (b) the gas pressure maps. Panels (c) and (d) plot gas pressure, temperature, and radiation loss along the selected field line crossing the blob center.
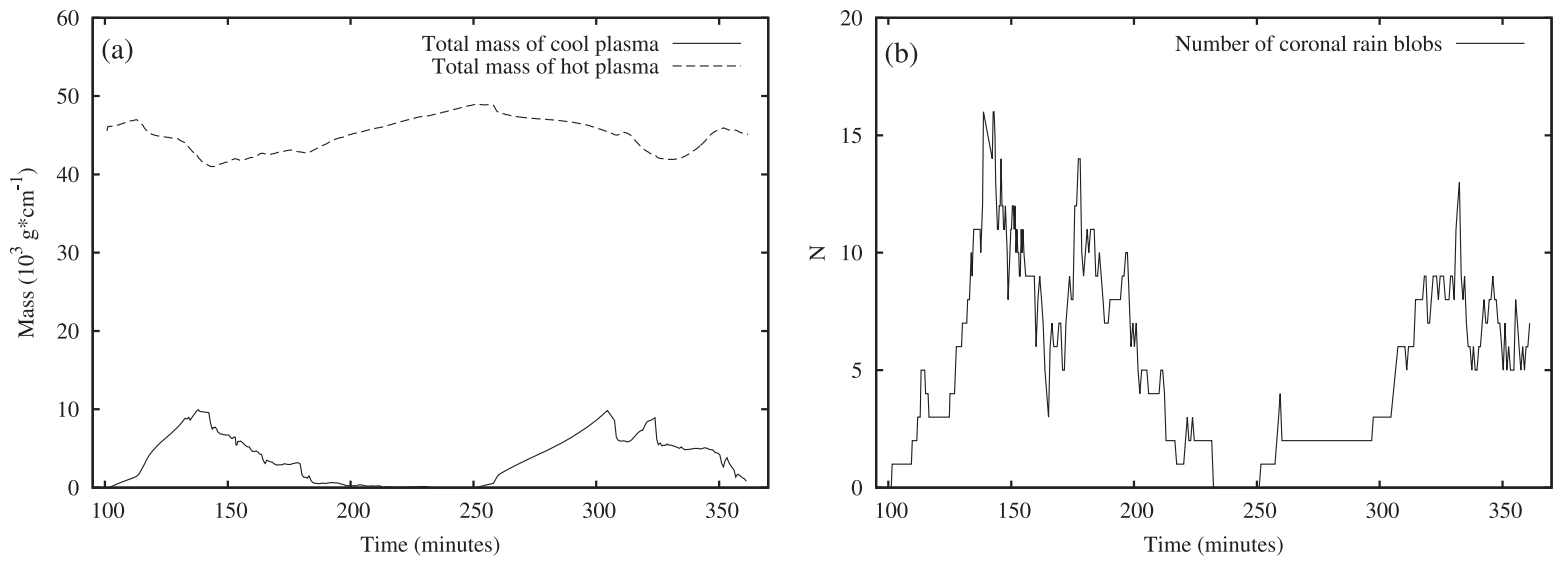

Figure 6. (a) Total mass of cool and of hot plasma in the corona vs. time. (b) Number of blobs vs. time.

Rouppe van der Voort 2012). This is the first time that we can report limit cycles of coronal rain in a multidimensional simulation, which confirms that constant heating conditions that provide enough energy can form secondary (or even more) coronal rain cycles in a single arcade. From panels (a) and (b) of Figure 6, we find the time interval between the first and secondary cycle to be around 175 minutes, when measured between successive maxima in cool mass matter. Panel (a) of Figure 6 shows the temporal evolution of total mass of hot coronal plasma which is the mass in the corona, excluding the cool plasma identified by the above criteria. We find that at $t \approx 140$ minutes, the total mass of cool plasma reaches its peak in the first cycle in panel (a) of Figure 6 , while at $t \approx 143$ minutes the catastrophic cooling process has cooled down most of the hot plasma in the corona shown in panel (a) of Figure 6. From about $t \approx 133$ minutes, blobs begin to fall into the TR, then the evaporation of plasma in the chromosphere driven by the extra heating $H_{1}$ fills the evacuated loops left by blobs that 
have already sank into the chromosphere. From this moment, until the onset of the secondary cycle of our coronal rain shower at $t \approx 250$ minutes, it takes about 120 minutes, which is of similar duration to the time for the first cycle to reach its onset (about 100 minutes). So although we infer from the total mass evolution of cool plasma in panel (a) that there is only about 50 minutes between the ending of the first and the beginning of the second cycle, actually the continued heating at the chromosphere has already spontaneously began to fill the empty corona 70 minutes before. We also see that the total mass of hot plasma before the onset of the secondary cycle is higher than in the first cycle (panel (a)), which leads to a longer lasting secondary cycle with more mass in condensations. Panel (a) of Figure 6 indicates that at $t \approx 130$ minutes (before the first blob falls into the chromosphere), there is at least $9 \times 10^{3} \mathrm{~g} \mathrm{~cm}^{-1}$ of cool plasma in the corona, which originally was hot plasma. Meanwhile panel (a) of Figure 6 also suggests that compared with the corona before the onset of catastrophic cooling at $t \approx 100$ minutes, the decrement in the same time of total mass of hot plasma at $t \approx 140$ minutes is only $5 \times 10^{3} \mathrm{~g} \mathrm{~cm}^{-1}$. The difference between the increase in cool plasma and the decrease in hot plasma indicates that during these 30 minutes since onset at $t \approx 100$ minutes, the evaporation in the chromosphere evaporates $4 \times 10^{3} \mathrm{~g} \mathrm{~cm}^{-1}$ into the corona, i.e., at an evaporation rate of $2.2 \mathrm{~g} \mathrm{~cm}^{-1} \mathrm{~s}^{-1}$. We can similarly estimate an evaporation rate of $2.3 \mathrm{~g} \mathrm{~cm}^{-1} \mathrm{~s}^{-1}$ between the onset of the secondary cycle and the moment its first blob falls into the TR. Until the onset of the first cycle at $t \approx 100$ minutes, the increment of total hot plasma from turning on the extra heating $H_{1}$ is about $13.2 \times 10^{3} \mathrm{~g} \mathrm{~cm}^{-1}$ in total, further confirming this evaporation rate of $2.2 \mathrm{~g} \mathrm{~cm}^{-1} \mathrm{~s}^{-1}$. Based on these estimates, we infer that anywhere in both simulated cycles the constant extra heating $H_{1}$ leads to a nearly constant evaporation rate. We can thus extrapolate to even more cycles expected further on, and interpret these limit cycles as a chronological sequence of mass recycling between chromosphere and corona: heating in the chromosphere brings plasma to the corona by evaporation, where it ultimately triggers catastrophic cooling; the cooling process manages the coronal plasma into coronal rain where the plasma drains back to the chromosphere; and persistent heating causes the chromospheric material to evaporate again toward the corona.

Although the duration and peak value of the total mass in both computed cycles are similar, their initial condensation rates (in contrast to the previously discussed evaporation rate) computed from the temporal variation of their total mass curve work out to be 6.7 and $4.5 \mathrm{~g} \mathrm{~cm}^{-1} \mathrm{~s}^{-1}$, respectively, and thus are different. It is known from linear thermal instability theory (Field 1965) and 1D simulation results in Xia et al. (2011) that this initial condensation rate in catastrophic cooling depends on parameters controlling the energy input from heating. One notices that the condensation rate (the local derivative of the solid curve in panel (a) of Figure 6) varies dramatically even within one cycle, despite a constant heating energy input in our multidimensional simulation. We now will interpret the reason for the changes seen in the condensation rate by surveying especially the process of growth for the first condensation, which forms under a relatively simple and almost symmetric condition.

The solid line in panel (a) of Figure 7 shows the temporal evolution of the mass accumulation for this first condensation (the one from Figure 2 ) from $t \approx 100$ to 110 minutes. Its nearly linear behavior quantifies that the condensation rate remains almost constant in this time interval at a value of about $2.3 \mathrm{~g} \mathrm{~cm}^{-1} \mathrm{~s}^{-1}$. We deliberately do not discuss what happens to the first condensation after $t \approx 110$ minutes, since afterwards it breaks into two smaller blobs. In the same figure, panel (a), the dashed line displays the growth of the total mass of cool plasma as seen on a single field line through the center of the first condensation, i.e., in a $1 \mathrm{D}$ fashion. To show this, we identify the group of grid points that are passed by the field line. The total mass of cool plasma determined on the single field line keeps growing in time, but its growth rate is much smaller than that for the whole 2D condensation. Panel (b) of Figure 7 quantifies the temporal evolution of typical lengths for the first condensation, where we quantify both the length parallel to the magnetic field lines and the length perpendicular to the magnetic field lines. This indicates that blob growth in the perpendicular direction is much faster than in the parallel one, which can be seen visually as well in all columns in Figure 2 and Figure 3. As discussed in Fang et al. (2013), the low pressure region surrounding the first condensation onset leads to magnetic restoring forces on adjacent loops. These in turn influence the location where the catastrophic cooling will take place on the adjacent loops, which are all close to the thermal instability onset. The different growth rates found for blob sizes in these two directions then relate to the fairly fast "growth" along the perpendicular direction due to sympathetic runaway cooling onset, versus the slower growth seen in the parallel one, which is the only one found in 1D setups. The average density of each cell of the condensation is quantified in panel (c) of Figure 7, and this density stays basically the same in the forming process, meaning that the total mass of the condensation is just proportional to the increasing number of neighboring grid cells that contain cool plasma. While the number of cells in the condensation increases in both directions, the larger condensation rate of the whole blob in panel (a) of Figure 7 again directly reflects the faster growth in size in the perpendicular direction. We conclude that the growth of the total mass of individual blobs in our simulation is mainly determined by the onset of catastrophic cooling in neighboring loops rather than the growth along the loops in which catastrophic cooling gets triggered. We can indeed verify this $2 \mathrm{D}$ growth aspect by further showing a correlation between the total mass of cool plasma and two other measures, which holds up for an even longer time than the first 10 minutes, i.e., when several blobs have started to form. This is shown in panel (d) in Figure 7 where we plot the temporal evolution of the total mass of cool plasma, the size of the onset TR, and the total blob region width. The total blob region width indicates the total width of all magnetic loops where catastrophic cooling takes place on. The size of the onset TR means the corresponding width as found at the TR height, of all the loops undergoing catastrophic cooling. Because of the magnetic arcade configuration adopted, these size measures for the affected loops give higher values for higher locations, i.e., the total blob region width always exceeds the (field aligned remapped) onset TR size. The latter size of the onset TR shows a nice correlation with the total mass of the cool plasma evolution.

\subsection{The Fate of Blobs Hitting the TR}

In the simulation, we observe plenty of blobs hitting the TR and disappearing into the lower chromosphere, which is also known to occur in observations (Antolin et al. 2010; Antolin \& 

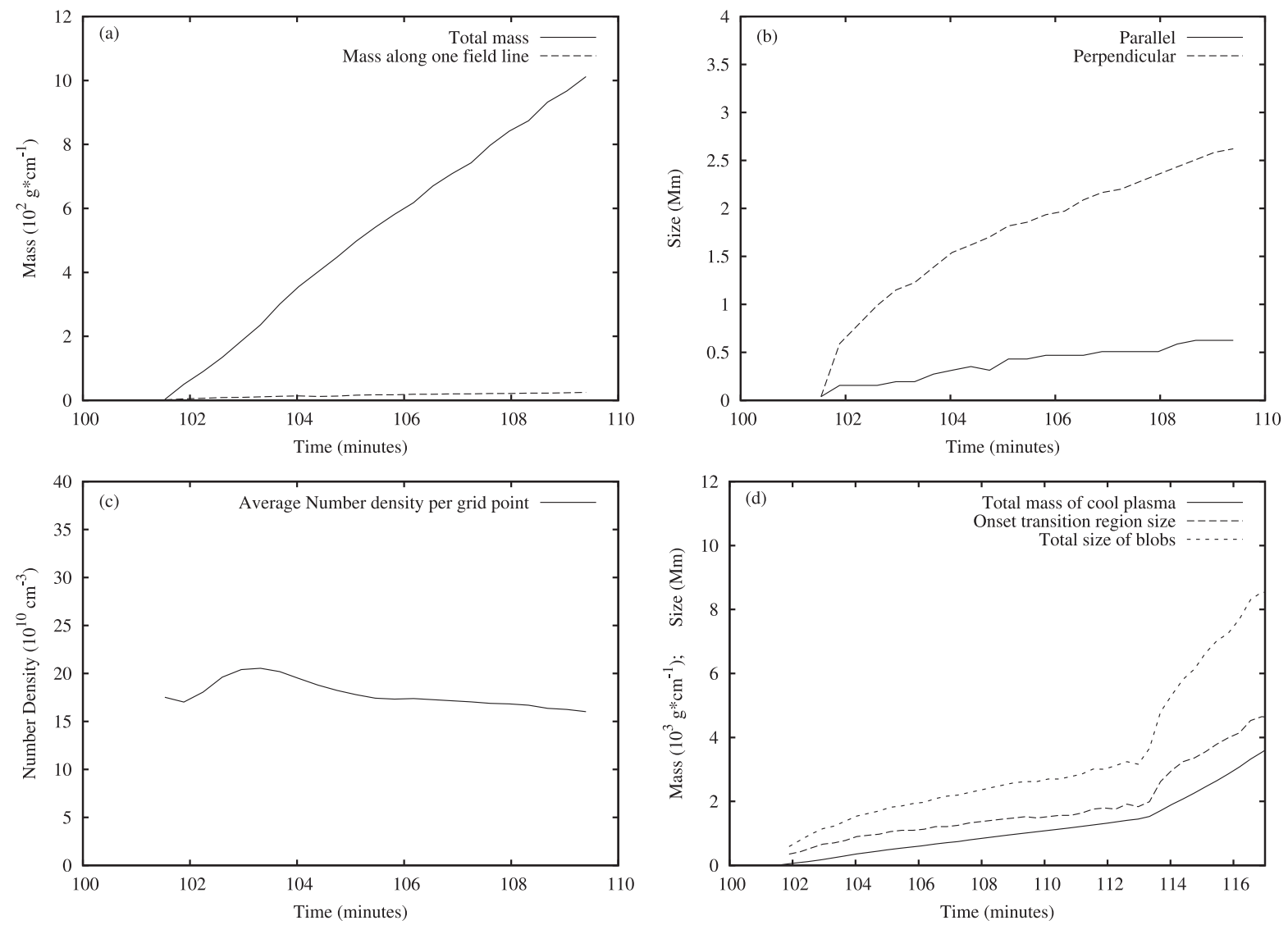

Figure 7. (a) Mass in the first condensation vs. time. The dashed line shows a measurement performed along one field line only. (b) Lengths vs. time of the first condensation (solid: length parallel to the magnetic field lines; dashed: length perpendicular to the magnetic field lines). (c) Average density evolution of the first condensation. (d)Total mass of cool plasma in the corona (solid), onset transition region size (long dashed) and total size of blobs (short dashed) vs. time within the range from 100 to 117 minutes.

Rouppe van der Voort 2012). Tripathi et al. (2009) observed high-speed downflows and concurrent upflows in coronal loops close to the footpoints and argue in favor of upflows in coronal loops at higher temperatures. Antolin et al. (2010) confirmed that the high-speed downflows represent the cool plasma, which correspond to the falling blobs in our simulation (see also the movie in Fang et al. 2013). Meanwhile, our 2.5D simulation also shows the possibility of triggering concurrent upflows as observed by Tripathi et al. (2009) and Kleint et al. (2014). Panel (a) in Figure 8 shows the number density map at $t \approx 143.7$ at a moment when falling blobs sink into the TR and compress the plasma on its way (at about $x \approx-2.1 \mathrm{Mm}$ ). Panel (b) in Figure 8 shows the vertical velocity map at the same location and instant, which clearly displays the concurrent upflows rising at the tails of the declining blobs in the same field line bundles. Hence, this answers the question in Kleint et al. (2014) whether the upflows can flow along the same field lines as the downflows. These upflows in our simulation are actually rebound shocks from the impact of the blobs on the TR. They arise immediately when the blobs impact on the TR and spread from one footpoint to another footpoint in around 5 minutes with a velocity of around $50 \mathrm{~km} \mathrm{~s}^{-1}$. From Panel (a) we can see the enhanced density left after the passage of these rebound shocks. However, panel (c) in Figure 8, which quantifies temperature, indicates that the temperature in the loop already increases before the rebound shocks have reached far into the loop, since the parametrized background heating $H_{0}$ very efficiently heats the low density loops left by falling blobs.
Panel $\mathrm{d}$ also shows the temperature, but now on a larger domain and at a later time. It shows that afterwards the rebound shocks heat the low density loops to an even higher temperature of $2.0 \mathrm{MK}$. After the rebound shocks reach the other footpoint, the loops are at high temperature of about $2 \mathrm{MK}$ but with a low number density of $1 \times 10^{8} \mathrm{~cm}^{-3}$. We distinguish this from further upflows coming from evaporation due to the extra strong heating $H_{1}$ located in the chromosphere. This enhances the density to $1 \times 10^{9} \mathrm{~cm}^{-3}$ again and the temperature to $2.3 \mathrm{MK}$. However, these upflows from evaporation rise with a much slower velocity of around $15 \mathrm{~km} \mathrm{~s}^{-1}$.

To quantify even further the detailed fate of a blob when it hits and descends into the TR, Figure 9 shows the temporal evolution of the mass, density, velocity, kinetic energy, momentum, and temperature of the first coronal rain blob to hit the TR from the corona and to sink down into the chromosphere. The vertical dashed line in each panel of Figure 9 points at $t \approx 132$ minutes when this blob hits the TR. Because the density and temperature of plasma in the TR is comparable with those of the blobs, we can no longer use only the density and temperature as criteria to distinguish blobs when they are near or partially below the TR. In order to identify plasma belonging to the blob as it hits and descends in the TR after $t \approx 132$ minutes, we change our criteria to require the local velocity to be larger than $3 \mathrm{~km} \mathrm{~s}^{-1}$ and the locations are below the TR line $y^{\text {tr }}(x, t)$ after $t \approx 132$ minutes. Since the velocity of the plasma in the TR is almost zero, this velocitybased criterion captures the location of sinking blobs. In panel 

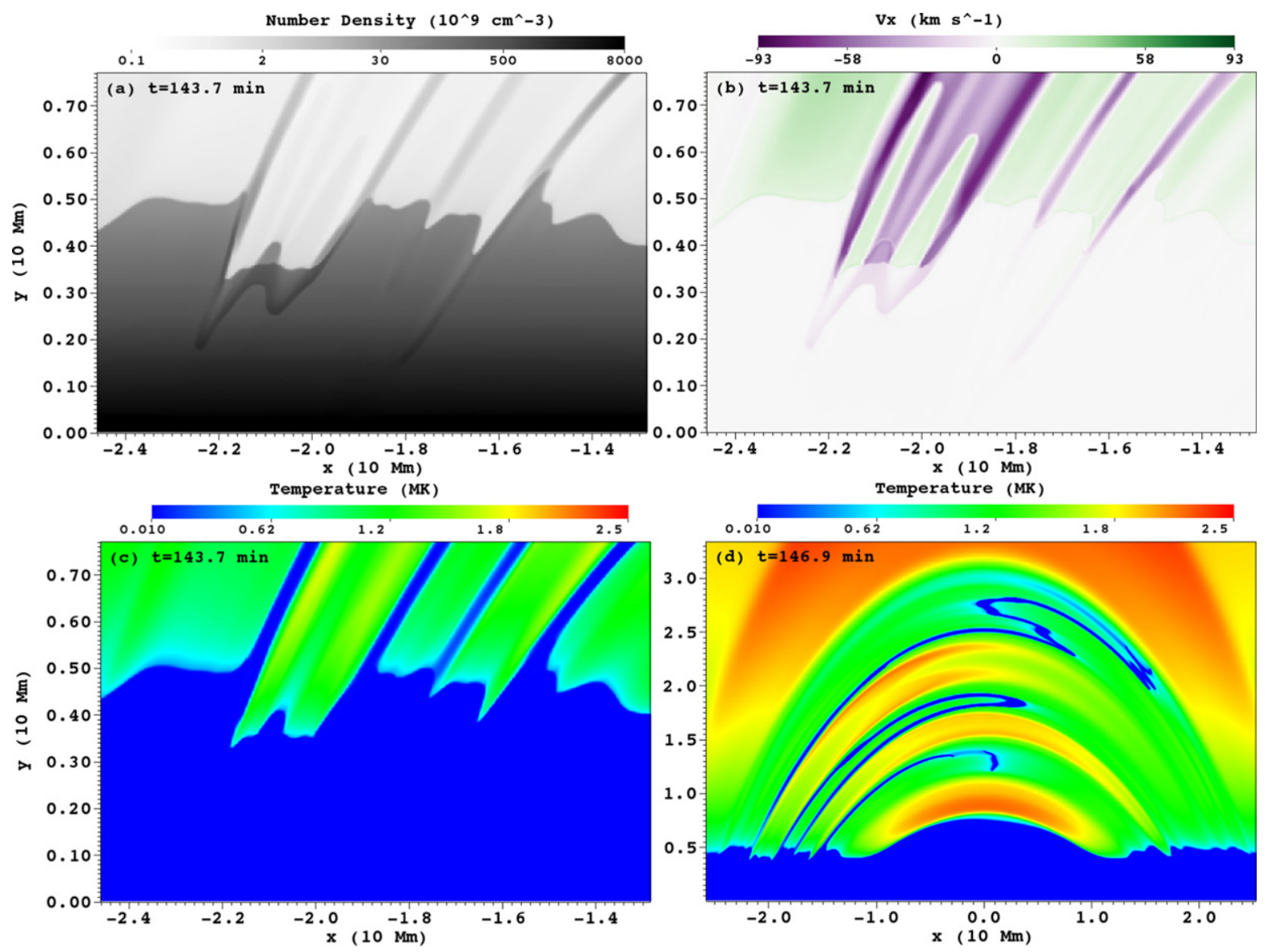

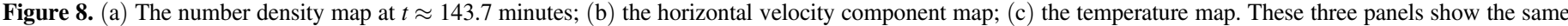

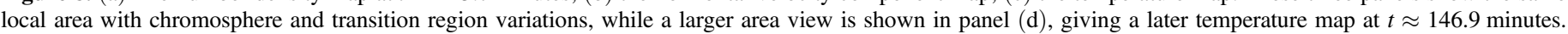

(a) of Figure 9, we find that the mass identified as blob material by the above criteria begins to increase at $t \approx 132.7$ minutes. This is because the mass detected not only includes the blob itself, but also counts mass compressed and accelerated by the blob impact. At $t \approx 136$ minutes, the total mass affected reaches its peak at six times the original blob mass. After $t \approx 136$ minutes, due to the combined influence of reflectiontransmission processes at the TR and the higher gas pressure from the impact, the velocities in much of the blob-impacted area decrease to values smaller than the criterion $3 \mathrm{~km} \mathrm{~s}^{-1}$. This is then seen as a mass decrease in our panel (a). In panel (b) of Figure 9, the density versus time profile keeps rising while the blob hits the TR. As we know, this blob impact compresses the TR plasma swept up by the blob and transfers momentum from the sinking blob to the impacted plasma, and therefore in panel (c) of Figure 9 we find that the average velocity of the region identified keeps decreasing during the whole process, as well as the kinetic energy shown in panel (d). Panel (e) of Figure 9 shows the total momentum of the mass identified. Due to the gravitational acceleration, the blob momentum keeps increasing until it reaches its maximum value at $t \approx 136$ minutes, then it reduces quickly. This is a combination of the mass evolution in panel (a) and the velocity information from panel (c). The momentum and velocity decreasing after the impact relate to momentum transfer to the surrounding TR and upper chromosphere plasma, until the regions selected by the velocity-based criteria vanishes: the local conditions settle to static chromosphere conditions. Panel (f) of Figure 9 shows the average temperature evolution during the blob impact. The temperature increases before hitting the TR due to the compressional heating when the blob descends through the higher gas pressure region just above the TR. After the impact, since also more cooler material gets identified as impacted matter, one settles back to upper chromospheric temperature values.

The impact speed of blobs in Figure 9 is around $30 \mathrm{~km} \mathrm{~s}^{-1}$, and the highest impact speed of all blobs in our simulation is around $60 \mathrm{~km} \mathrm{~s}^{-1}$ and number densities range from 4 to $6 \times 10^{10} \mathrm{~cm}^{-3}$. Our maximum impact speed is much lower than the falling speeds reported in Kleint et al. (2014) which went up to $200 \mathrm{~km} \mathrm{~s}^{-1}$. They report that these coronal rain events with high impact speeds are correlated with local brightenings which probably indicate an increase of density and temperature in the TR. Panel (b) of Figure 9 and panel (a) of Figure 8 confirm the expected increase of the number density of impacts in our simulation.

\subsection{Counter-streaming Flows}

We also find another interesting phenomenon in our numerical simulation, namely the self-consistent establishment of counter-streaming flows. Such anti-parallel flows are very commonly found in solar observations, especially in prominences (Alexander et al. 2013). Panels (a), (b), and (c) in Figure 10 respectively show the signed velocity magnitude map (with the sign taken from the horizontal velocity component), the gas pressure map, and the number density 

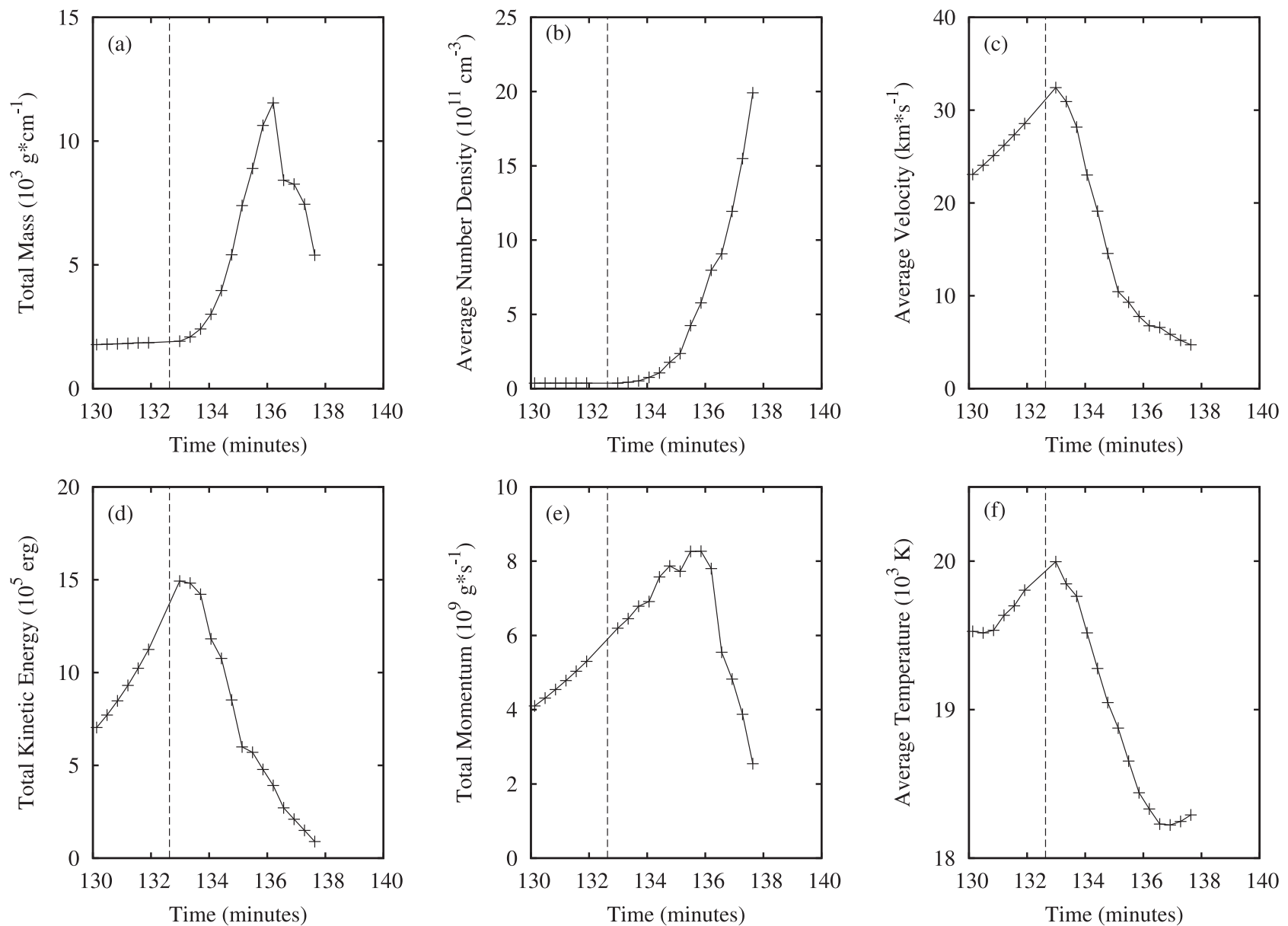

Figure 9. Total mass, average number density, average velocity, total kinetic energy, total momentum and temperature evolution of the blob, which first impacts and sinks into the transition region during this time period. The vertical dashed line indicates the time when this blob hits the transition region.

map at $t \approx 132.6$ minutes. Panel (d) shows the signed velocity magnitude map as in panel (a), but at a later time, namely at $t \approx 146.9$ minutes. These four panels in Figure 10 display many cases of counter-streaming flows established on neighboring field line bundles in our simulation and allow the origins of counter-streaming flows to be explained. After thermal instability inducing a runaway catastrophic cooling and initial growth in an almost static state, the condensations lose their delicate force balance and begin to slide toward one footpoint along magnetic field lines. Whether a particular condensation segment slides to the left or right is influenced by its initial location and local total force balance (gravity, gas pressure gradient, and magnetic field force). Once in motion, they are accelerated by the field-projected gravitational force. Meanwhile catastrophic cooling keeps taking place around the condensations. As discussed in Section 3.1, the initial catastrophic cooling process depletes the local plasma and sucks in fast inflows, then the spontaneous rebound shocks heat the plasma and increase the gas pressure. Afterwards, no stronger inflows are driven again due to the increased gas pressure. However, there can be several blob pairs lying in the same or neighboring field line bundles, as shown in panel (c) of Figure 10. Figure 11 shows gas pressure maps with magnetic field lines at $t \approx 109.4$ and 113.0 minutes. The black contour relates to the temperature distribution and is an isocontour at $0.1 \mathrm{MK}$. Both the gas pressure and temperature in panel (a) in
Figure 11 indicate the clear PCTR around the blob as previously discussed in Figure 5. After 3 minutes, panel (b) of Figure 11 shows two low (white) pressure sections after the blob breaks into three segments. These low pressure sections slant through the field lines and they are the elongated PCTR cross sections from the original parts of the whole blob in panel (a) of Figure 11. Because of the strong radiation in the PCTR, the temperatures inside these elongated regions remain low during their deformation. As a result, we could consider these cross sections to undergo isothermal expansion. Because the condensed mass in these narrow regions grows much slower than their areal growth due to elongation, the densities inside these elongated cross sections decrease faster as well as the gas pressure. This leads to blob sequences with low pressure sections in between them. This is also seen in panel (b) of Figure 10 where a sequence of blobs show up with low (white) pressure sections in between them. The depleted areas trigger siphon inflows that refill these regions. Then this pair of siphoned fast inflows establish the counter-streaming flows between the pair of neighboring blobs. Panels (a) and (b) in Figure 10 also show that falling condensations with larger velocities induce larger density depletions and lower gas pressure areas on their way down, which lead to faster inflows than those found for more static condensations.

Panel (d) in Figure 10 indicates a different origin of counterstreaming flows at $t \approx 146.9$ minutes. As we discuss in 

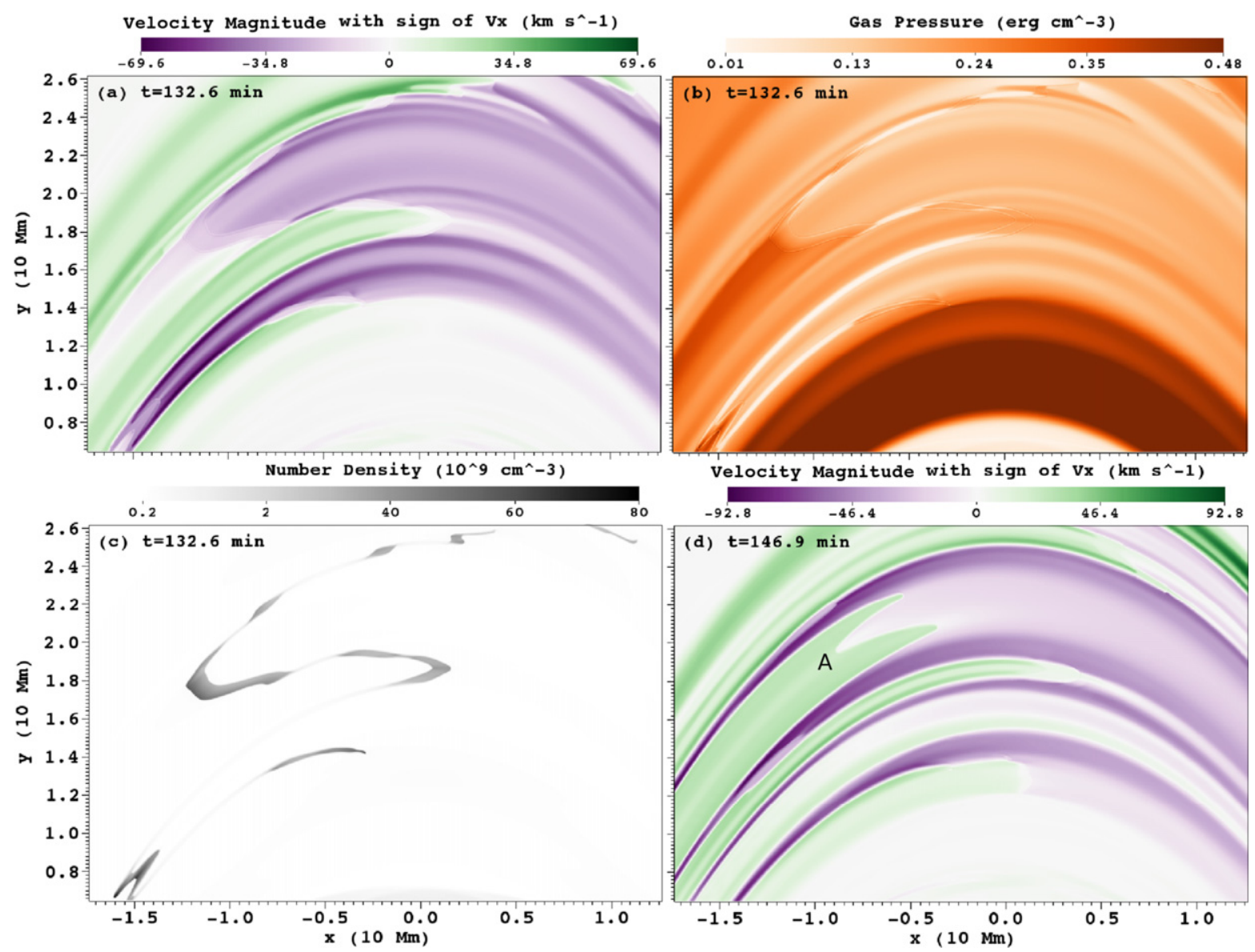

Figure 10. (a) Velocity magnitude map signed with horizontal velocity component at $t \approx 132.6$ minutes. Panel (b) shows the gas pressure map and panel (c) shows the number density map at the same time. In panel $(\mathrm{d})$, the signed velocity magnitude map is shown later at $t \approx 146.9$ minutes, where the label A points to the upflows resulting from the rebound event shown in detail in Figure 8.

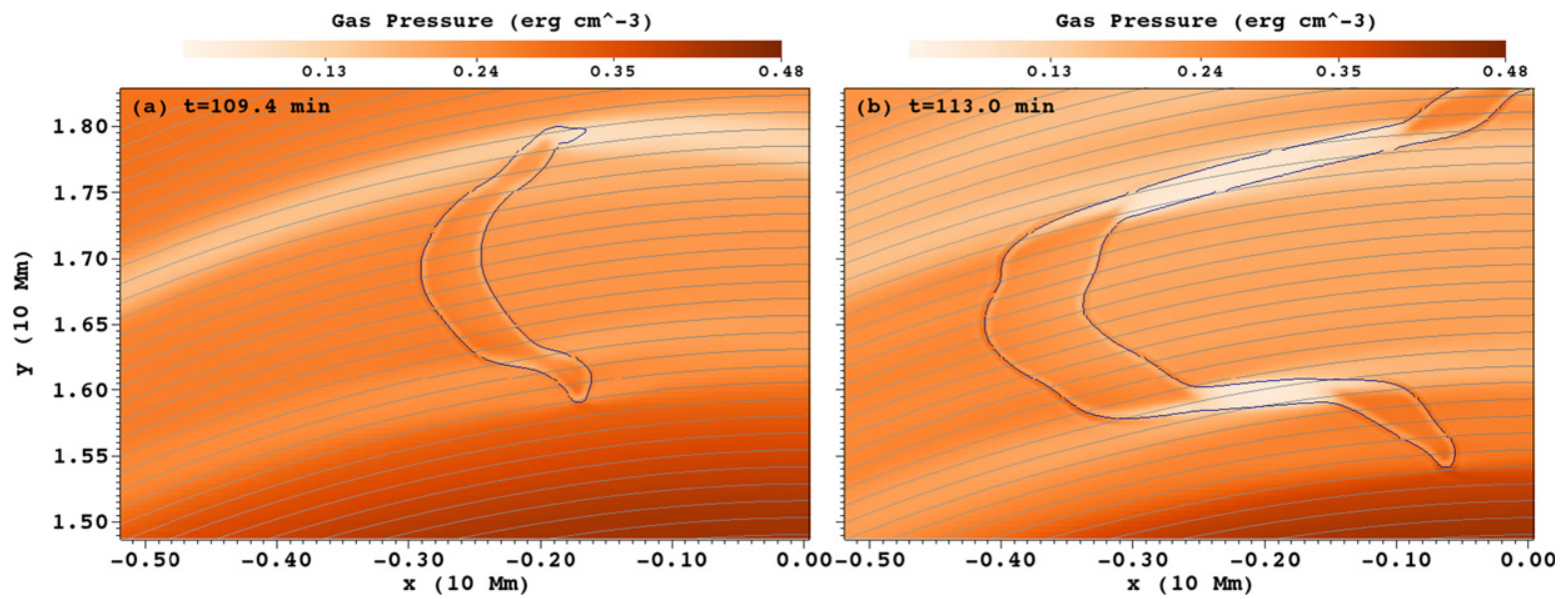

Figure 11. Panels (a) and (b) show the gas pressure maps with magnetic field lines at $t \approx 109.4$ and 113.0 minutes. The black contour relates to the temperature distribution with level at $0.1 \mathrm{MK}$.

Section 3.3, we observe that after blobs decline into the TR, concurrent upflows rise up toward the loop apex. Upflows labeled A in panel (d) in Figure 10 are the concurrent upflows shown in panel (b) of Figure 8, but about 3 minutes later (concurrent with the later temperature panel (d) of Figure 8). Upflows arising from blob impacts also have the chance to establish a counter-streaming flow if there is an opposite flow pattern in the neighboring loops. The difference between these two different origins for counter-streaming flows is that the one based on depleted sections between a pair of blobs lying on neighboring field line bundles can last through the whole falling process of blobs, or on timescales of about 10 minutes, while the other ones will vanish after the upflows refill the loops, typically in a shorter timescale of about 5 minutes. 

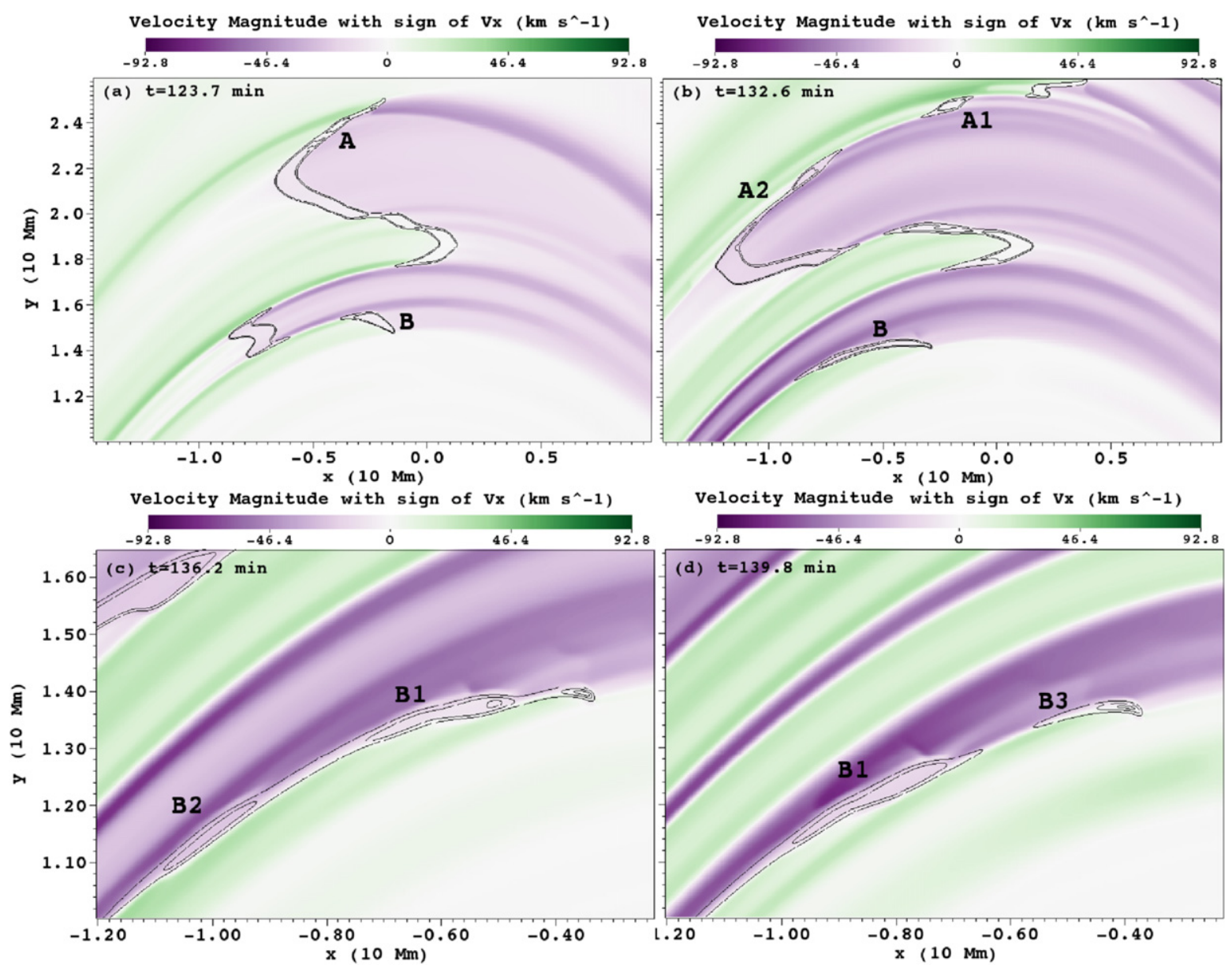

Figure 12. Color maps show the velocity magnitude map with the sign of the horizontal velocity component at $t \approx 123.7$ (a), 132.6 (b), 136.2 (c), and 139.8 (d) minutes. The black contours relate to the number density distribution with levels at 7, 25, and $50 \times 10^{9} \mathrm{~cm}^{-3}$. This clearly shows how shear flow effects induce blob fragmentation and evolution.

\subsection{Shear Flow Effects}

The sheared flows that are established by the detailed blob dynamics could also in return influence the further evolution of the condensations. An example is shown in panel (a) of Figure 12, where we show a signed velocity map, with overlaid contours of the density distribution of condensations at levels of 7, 25, and $50 \times 10^{9} \mathrm{~cm}^{-3}$ at $t \approx 123.7$ minutes. Concentrating on the density feature labeled A, after its initial formation, sheared flows already begin to take shape. About 10 minutes later, this segment $\mathrm{A}$ is seen as segment $\mathrm{A} 1$ and $\mathrm{A} 2$ in panel (b) of Figure 12, and the condensation has broken into two distinct segments with increasing separation between them. Segment $\mathrm{A} 2$ is also going to break into two segments a little later. At $t \approx$ 123.7 minutes in panel (a) of Figure 12, this segment A feature is more like one whole elongated condensation. However, by $t \approx 132.6$ minutes in panel (b) in Figure 12 , several condensations behave totally separately from each other. Another example is the one of segment B in panel (a) and panel (b). This breaks up into segment B1 and segment B2 in panel (c) at $t \approx 136.2$ minutes. Then segment B1 further breaks into segment $\mathrm{B} 1$ and $\mathrm{B} 3$ in panel $(\mathrm{d})$ at $t \approx 139.8$ minutes. This gradual change from one elongated dense blob or strand breaking into several segments, surrounded by fast sheared flows, hints at the influence of Kelvin-Helmholtz instabilities $(\mathrm{KHI})$. However, there is no clear vorticity pattern emerging in our simulation that would clearly identify KHI development, which may not have enough time to develop. We speculate that other KHI-related substructure may well arise under different parameter settings (field strength, heating scale height), but already establish that sheared flows contribute to the breaking up of elongated condensations into smaller fragments.

\section{CONCLUSIONS}

We extended our earlier multidimensional, magnetohydrodynamic simulations of coronal rain occurring in magnetic arcades hosting chromospheric, TR, and coronal plasma. The new main results can be summarized as follows.

1. We find that after the initial formation stage of condensations, expansion rebound shock fronts introduced by fast siphon inflows typically display a fanshaped structure. The local conditions of where condensations form influences the detailed dynamics and expansion of these rebound shocks, and can lead to asymmetric expansion fronts or only one-sided expansion shock fronts.

2. We discussed the process of establishing a structured PCTR around coronal rain condensations. The strong radiation loss at the boundary of blobs results in local 
dips in the gas pressure structure at the blob boundary where the temperature rises sharply from $0.01 \mathrm{MK}$ to a coronal temperature of $0.5 \mathrm{MK}$.

3. By extending our $2.5 \mathrm{D}$ simulation to a longer time of $6 \mathrm{hr}$, we obtain a secondary cycle of coronal rain in the simulation. This secondary cycle confirms the deductions from previous 1D simulations and observations that by providing consistent and enough energy, coronal rain can form a secondary cycle or even more.

4. We study the condensation rate in our 2.5D simulation and find the growth of cool mass in the corona to show a good correlation especially with the faster growth rate in the length of condensations in the direction perpendicular to the field lines. This indicates that the growth of cool mass is dominated by the onset of runaway cooling in neighboring loops. This significantly exceeds the rates obtained in studies of this growth rate in 1D models, purely along field lines, as we also need to understand the expansion speed of onset of runaway cooling in neighboring loops. By performing detailed quantitative analysis, we also find that no matter what happens in the corona, constant heating in the chromosphere keeps on evaporating a certain amount of hot plasma into the corona, establishing a mass cycle.

5. We look into the impact of declining blobs on the TR, and find that their rebound shocks can spread as upflows from one footpoint to another footpoint. Following the rebound shocks, evaporation driven upflows with a slower velocity refill the loops and heat them to $2.3 \mathrm{MK}$ again.

6. Plenty of counter-streaming flows are found in our simulation, and we demonstrated several reasons for forming these flows. One is that the extremely low gas pressure area between two neighboring coronal rain blobs drives strong siphon flows toward it. These shear flows accompany the blobs until they fall into the TR.

7. The counter-streaming flows also in return influence the deformation of the blobs, which can break into several segments, starting from an elongated one.

The research has been sponsored by an Odysseus grant of the FWO Vlaanderen. The results were obtained in the KU Leuven GOA project GOA/2015-014 and by the Interuniversity Attraction Poles Programme initiated by the Belgian Science Policy Office (IAP P7/08 CHARM). Part of the simulations used the infrastructure of the VSC-Flemish Supercomputer Center, funded by the Hercules Foundation and the Flemish Government-Department EWI. We acknowledge fruitful discussions with P. Antolin, and a very helpful referee report. The work is supported by International Programme "Implications for coronal heating and magnetic fields from coronal rain observations and modeling" of the International Space Science Institute (ISSI), Bern.

\section{REFERENCES}

Alexander, C. E., Walsh, R. W., Régnier, S., et al. 2013, ApJL, 775, L32

Antiochos, S. K., \& Klimchuk, J. A. 1991, ApJ, 378, 372

Antiochos, S. K., MacNeice, P. J., Spicer, D. S., \& Klimchuk, J. A. 1999, ApJ, 512, 985

Antiochos, S. K., MacNeice, P. J., \& Spicer, D. S. 2000, ApJ, 536, 494

Antolin, P., \& Rouppe van der Voort, L. 2012, ApJ, 745, 152

Antolin, P., Shibata, K., \& Vissers, G. 2010, ApJ, 716, 154

Beckers, J. M. 1962, AuJPh, 15, 327

Cada, M., \& Torrilhon, M. 2009, JCoPh, 228, 4118

Chae, J., Wang, H., Qiu, J., et al. 2001, ApJ, 560, 476

Colgan, J., Abdallah, J., Jr., Sherrill, M. E., et al. 2008, ApJ, 689, 585

Dahlburg, R. B., Antiochos, S. K., \& Klimchuk, J. A. 1998, ApJ, 495, 485

De Groof, A., Bastiaensen, C., Müller, D. A. N., Berghmans, D., \& Poedts, S. 2005, A\&A, 443, 319

De Groof, A., Berghmans, D., van Driel-Gesztelyi, L., \& Poedts, S. 2004, A\&A, 415, 1141

Fang, X., Xia, C., \& Keppens, R. 2013, ApJL, 771, L29

Field, G. B. 1965, ApJ, 142, 531

Fontenla, J. M., Avrett, E. H., \& Loeser, R. 1991, ApJ, 377, 712

Goldsmith, D. W. 1971, SoPh, 19, 86

Hildner, E. 1974, SoPh, 35, 123

Kamio, S., Peter, H., Curdt, W., \& Solanki, S. K. 2011, A\&A, 532, A96

Karpen, J. T., \& Antiochos, S. K. 2008, ApJ, 676, 658

Karpen, J. T., Antiochos, S. K., Hohensee, M., Klimchuk, J. A., \& MacNeice, P. J. 2001, ApJL, 553, L85

Karpen, J. T., Antiochos, S. K., \& Klimchuk, J. A. 2006, ApJ, 637, 531

Karpen, J. T., Antiochos, S. K., Klimchuk, J. A., \& MacNeice, P. J. 2003, ApJ, 593,1187

Karpen, J. T., Tanner, S. E. M., Antiochos, S. K., \& DeVore, C. R. 2005, ApJ, 635,1319

Kawaguchi, I. 1970, PASJ, 22, 405

Keppens, R., Meliani, Z., van Marle, A. J., et al. 2012, JCP, 231, 718

Keppens, R., \& Porth, O. 2014, JCoAm, 266, 87

Keppens, R., \& Xia, C. 2014, ApJ, 789, 22

Kleint, L., Antolin, P., Tian, H., et al. 2014, ApJL, 789, L42

Levine, R. H., \& Withbroe, G. L. 1977, SoPh, 51, 83

Leroy, J.-L. 1972, SoPh, 25, 413

Luna, M., Karpen, J. T., \& DeVore, C. R. 2012, ApJ, 746, 30

Meliani, Z., Keppens, R., \& Giacomazzo, B. 2008, A\&A, 491, 321

Mendoza-Briceño, C. A., Sigalotti, L. D. G., \& Erdélyi, R. 2005, ApJ, 624, 1080

Mok, Y., Drake, J. F., Schnack, D. D., \& van Hoven, G. 1990, ApJ, 359, 228

Mok, Y., Mikić, Z., Lionello, R., \& Linker, J. A. 2005, ApJ, 621, 1098

Mok, Y., Mikić, Z., Lionello, R., \& Linker, J. A. 2008, ApJL, 679, L161

Müller, D. A. N., De Groof, A., Hansteen, V. H., \& Peter, H. 2005, A\&A, 436, 1067

Müller, D. A. N., Hansteen, V. H., \& Peter, H. 2003, A\&A, 411, 605

Müller, D. A. N., Peter, H., \& Hansteen, V. H. 2004, A\&A, 424, 289

O'Shea, E., Banerjee, D., \& Doyle, J. G. 2007, A\&A, 475, L25

Parker, E. N. 1953, ApJ, 117, 431

Porth, O., Xia, C., Hendrix, T., Moschou, S. P., \& Keppens, R. 2014, ApJS, 214,4

Schrijver, C. J. 2001, SoPh, 198, 325

Scullion, E., Rouppe van der Voort, L., Wedemeyer, S., \& Antolin, P. 2014, ApJ, 797, 36

Townsend, R. H. D. 2009, ApJS, 181, 391

Tripathi, D., Mason, H. E., Dwivedi, B. N., del Zanna, G., \& Young, P. R. 2009, ApJ, 694, 1256

van Marle, A. J., Meliani, Z., Keppens, R., \& Decin, L. 2011, ApJL, 734, L26

Xia, C., Chen, P. F., Keppens, R., \& van Marle, A. J. 2011, ApJ, 737, 27

Xia, C., Chen, P. F., \& Keppens, R. 2012, ApJL, 748, L26

Wang, Y.-M. 1999, ApJL, 520, L71 